\title{
Efficient implementation of the invariant imbedding T-matrix method and the separation of variables method applied to large nonspherical inhomogeneous particles
}

\author{
Lei Bi ${ }^{a}$, Ping Yang ${ }^{a, *}$, George W. Kattawar ${ }^{b}$, Michael I. Mishchenko ${ }^{c}$ \\ a Department of Atmospheric Sciences, Texas A\&M University, College Station, TX 77843, USA \\ ${ }^{\mathrm{b}}$ Department of Physics \&' Astronomy, Texas AEM University, College Station, TX 77843, USA \\ ${ }^{\mathrm{c}}$ NASA Goddard Institute for Space Studies, 2880 Broadway, New York, NY 10025, USA
}

\section{A R T I C L E I N F O}

\section{Article history:}

Received 31 August 2012

Received in revised form

6 November 2012

Accepted 8 November 2012

Available online 23 November 2012

Keywords:

Invariant imbedding method

Separation of variables method

T-matrix

\begin{abstract}
A B S T R A C T
Three terms, "Waterman's T-matrix method", "extended boundary condition method (EBCM)", and "null field method", have been interchangeable in the literature to indicate a method based on surface integral equations to calculate the T-matrix. Unlike the previous method, the invariant imbedding method (IIM) calculates the T-matrix by the use of a volume integral equation. In addition, the standard separation of variables method (SOV) can be applied to compute the T-matrix of a sphere centered at the origin of the coordinate system and having a maximal radius such that the sphere remains inscribed within a nonspherical particle. This study explores the feasibility of a numerical combination of the IIM and the SOV, hereafter referred to as the IIM+SOV method, for computing the single-scattering properties of nonspherical dielectric particles, which are, in general, inhomogeneous. The IIM + SOV method is shown to be capable of solving light-scattering problems for large nonspherical particles where the standard EBCM fails to converge. The IIM+SOV method is flexible and applicable to inhomogeneous particles and aggregated nonspherical particles (overlapped circumscribed spheres) representing a challenge to the standard superposition T-matrix method. The IIM+SOV computational program, developed in this study, is validated against EBCM simulated spheroid and cylinder cases with excellent numerical agreement (up to four decimal places). In addition, solutions for cylinders with large aspect ratios, inhomogeneous particles, and two-particle systems are compared with results from discrete dipole approximation (DDA) computations, and comparisons with the improved geometric-optics method (IGOM) are found to be quite encouraging.
\end{abstract}

(c) 2012 Elsevier Ltd. All rights reserved.

\section{Introduction}

In various scientific disciplines (bio-optics, photonics, astrophysics, and atmospheric radiative transfer and remote sensing), accurate and efficient computations of the optical properties of dielectric particles are often

\footnotetext{
${ }^{*}$ Correspondence to: Department of Atmospheric Sciences, Texas A\&M University, TAMU-3150, College Station, TX 77843, USA.

E-mail address: pyang@tamu.edu (P. Yang).
}

required. The Lorenz-Mie theory and its modifications [1-5] applicable to homogenous or layered spheres cannot be used to compute the optical properties of morphologically complex particulates. Great strides have been made toward accurate simulations of the scattering of light by particles of various shapes and/or chemical compositions, but, although a variety of methods have been developed [6,7], each method has its own strengths and weaknesses. For example, the full-electromagnetic wave methods of solving Maxwell's equations become inefficient or even inapplicable when the particle size is excessively large, 
whereas the semi-empirical geometric-optics method is applicable to large particles but fails for small particles when the "ray" concept is not valid. Tremendous effort has been expended to broaden the computational domain of the existing light scattering computational methods e.g., [8-16] and to gain a better understanding of the singlescattering properties of nonspherical particles with size parameters ranging from the Rayleigh to geometric-optics regimes.

Waterman's T-matrix method (TMM) $[17,18]$ is an accurate and powerful tool capable of yielding a highly accurate numerical solution for the scattering of light by nonspherical particles [19-22]. In the literature, the technique is sometimes referred to as either the extended boundary condition method (EBCM) or the null field method. In contrast to many numerical techniques that explicitly consider the particle orientation and polarization state of the incident light in the simulations [23-28], the TMM calculates the T-matrix, a quantity independent of the propagation direction, incident light polarization state, and the scattering direction, and which allows for efficient computation of the orientation-averaged optical properties [29]. The conceptual framework of the TMM has subsequently been expanded to handle composite particles, layered particles, and more complicated scattering cases e.g., $[21,22,30-33]$. These developments have demonstrated the EBCM to be just one possible path to compute the T-matrix of the scattering object. Some attempts [8,34-37] to improve the limited applicability of the EBCM to certain particles have focused on numerical instability, convergence issues, and loss of precision; however, the maximum size parameter value for a convergent EBCM solution strongly depends on the particle shape. Specific details of various TMM implementations and relevant applications can be found in the texts [20-22] and a reference database $[38,39]$. The cumulative body of relevant research has made the TMM one of the most widely used approaches to obtain highly accurate numerical optical properties of morphologically complex particles with moderate aspect ratios and size parameters ranging from zero to $\sim 200$.

As mentioned, the T-matrix, relating the incident to the scattered field expansions in vector spherical wave functions (VSWFs), can be computed from several alternative approaches in addition to the EBCM. Johnson [40] derived the T-matrix from the standard electromagnetic volume integral equation (VIE) and developed an invariant imbedding method (IIM) to iteratively calculate the T-matrix. Schulz et al. [41] obtained the T-matrix of spheroids based on the separation of variables (SOV) method in spheroidal coordinates. A discrete dipole moment method to calculate the T-matrix was developed by Mackowski [42], while Loke et al. [43] incorporated the discrete dipole approximation (DDA) into the pointmatching method to calculate the T-matrix. A superposition T-matrix method (STMM) for multiple-sphere clusters developed by Peterson and Ström [30] and Mackowski and Mishchenko [44] is based on the addition translation theorem for vector spherical wave functions (VSWFs). In principle, any computational method that solves Maxwell's equations can be employed to calculate the T-matrix, although the computational efficiency, the computer memory requirements, the complexity of the numerical implementation, and the range of practical applicability can be quite variable.

The IIM for the calculation of the T-matrix has drawn scant attention since Johnson's study [40] (with specific applications to relatively small particles) was published in 1988. For example, the IIM is referenced neither in the T-matrix books [20-22] nor in the established T-matrix reference database $[38,39]$. Moreover, according to the ISI Web of Knowledge, Ref. [40] has previously been cited only 7 times. The use of the IIM in T-matrix calculations can be traced to its application to scattering problems in quantum mechanics [45]. Note that in addition to its application to the solution of Maxwell's equations, the IIM has been applied to the solution of the radiative transfer equation [46]. During the 1990s and early 2000s, significant advancement in relevant numerical techniques and computer resources has been made, and we believe the time has come to revisit the IIM for state-of-the-art numerical implementations.

This study explores the application of a numerical combination of Johnson's IIM method and the SOV (hereafter, IIM+SOV) to light scattering by large nonspherical and inhomogeneous particles. The remainder of the paper is organized as follows: Section 2 outlines the fundamentals of the T-matrix method and the invariant imbedding procedure; Section 3 includes the implementation of the IIM+SOV method to simulate light scattering by representative nonspherical and inhomogeneous particles, and both validates the accuracy and illustrates the efficiency by comparing IIM+SOV method results versus their counterparts computed from other methods; and, Section 4 summarizes our study.

\section{Theoretical basis}

\subsection{The T-matrix}

To elaborate the concept of the IIM for the computation of the T-matrix, we begin with the definition of the T-matrix based on the expansion of the incident and scattered fields in terms of VSWFs. The definition of the T-matrix depends on the adopted functional basis. To facilitate a combination of the T-matrix computation and the analytical orientationaverage algorithm outlined in Mishchenko et al. [21], we adopt the VSWFs in the exponential form in spherical coordinates. Let us consider the scattering of a plane electromagnetic wave $\overline{\mathbf{E}}^{i n c}(\mathbf{r})$ by a finite volume with a non-unity relative refractive index surrounded by an infinite homogeneous, isotropic, and non-absorbing host medium. We expand the incident and scattered fields in terms of VSWFs as:

$$
\begin{array}{ll}
\overline{\mathbf{E}}^{i n c}(\mathbf{r})=\sum_{n=1}^{\infty} \sum_{m=-n}^{n} \overline{\overline{\mathbf{Y}}}_{m n}(\theta, \phi) \overline{\overline{\mathbf{J}}}_{n}(r)\left[\begin{array}{l}
a_{m n} \\
b_{m n}
\end{array}\right], & \\
\overline{\mathbf{E}}^{s c a}(\mathbf{r})=\sum_{n=1}^{\infty} \sum_{m=-n}^{n} \overline{\overline{\mathbf{Y}}}_{m n}(\theta, \phi) \overline{\mathbf{H}}_{n}(r)\left[\begin{array}{l}
p_{m n} \\
q_{m n}
\end{array}\right], \quad r>r_{>},
\end{array}
$$

where $r_{>}$is the radius of the smallest circumscribed sphere of the scattering volume centered at the origin of 
the coordinate system and the angular function $\overline{\overline{\mathbf{Y}}}_{m n}(\theta, \phi)$ is a $3 \times 3$ matrix given by

$$
\begin{aligned}
& \overline{\mathbf{Y}}_{m n}(\theta, \phi)=(-1)^{m}\left[\frac{2 n+1}{4 \pi n(n+1)}\right]^{1 / 2} \\
& \quad \times e^{i m \phi}\left[\begin{array}{ccc}
0 & 0 & \sqrt{n(n+1)} d_{0 m}^{n}(\theta) \\
i \pi_{m n}(\theta) & \tau_{m n}(\theta) & 0 \\
-\tau_{m n}(\theta) & i \pi_{m n}(\theta) & 0
\end{array}\right],
\end{aligned}
$$

where $d_{0 m}^{n}(\theta)$ is the Wigner $d$ function, while $\pi_{m n}$ and $\tau_{m n}$ are given, respectively, by

$$
\begin{aligned}
& \pi_{m n}(\theta)=\frac{m}{\sin \theta} d_{0 m}^{n}(\theta)=\frac{1}{2} \sqrt{n(n+1)}\left[d_{1 m}^{n}(\theta)+d_{-1 m}^{n}(\theta)\right], \\
& \tau_{m n}(\theta)=\frac{d}{d \theta} d_{0 m}^{n}(\theta)=\frac{1}{2} \sqrt{n(n+1)}\left[d_{1 m}^{n}(\theta)-d_{-1 m}^{n}(\theta)\right] .
\end{aligned}
$$

The radial functions $\overline{\overline{\mathbf{J}}}_{n}(r)$ and $\overline{\mathbf{H}}_{n}(r)$ in Eqs. (1) and (2) are $3 \times 2$ matrices, given by

$$
\begin{aligned}
& \overline{\mathbf{J}}_{n}=\left[\begin{array}{ll}
j_{n}(k r) & 0 \\
0 & \frac{1}{k r} \frac{\partial}{\partial r}\left[r j_{n}(k r)\right] \\
0 & \sqrt{n(n+1)} j_{n}(k r) / k r
\end{array}\right], \\
& \overline{\overline{\mathbf{H}}}_{n}=\left[\begin{array}{ll}
h_{n}^{(1)}(k r) & 0 \\
0 & \frac{1}{k r} \frac{\partial}{\partial r}\left[r h_{n}^{(1)}(k r)\right] \\
0 & \sqrt{n(n+1)} h_{n}^{(1)}(k r) / k r
\end{array}\right],
\end{aligned}
$$

where $k$ is the wavenumber, $j_{n}(k r)$ is the spherical Bessel function of the first kind, and $h_{n}^{(1)}(k r)$ is the spherical Hankel function of the first kind. The following relations hold:

$\overline{\mathbf{Y}}_{m n} \overline{\overline{\mathbf{J}}}_{n}=\left(\operatorname{Rg} \overline{\mathbf{M}}_{m n}, \operatorname{Rg} \overline{\mathbf{N}}_{m n}\right), \overline{\mathbf{Y}}_{m n} \overline{\overline{\mathbf{H}}}_{n}=\left(\overline{\mathbf{M}}_{m n}, \overline{\mathbf{N}}_{m n}\right)$,

where $\overline{\mathbf{M}}_{m n}$ and $\overline{\mathbf{N}}_{m n}$ are column vector spherical wave functions that satisfy the radiation condition at infinity, while $\operatorname{Rg} \overline{\mathbf{M}}_{m n}$ and $\operatorname{Rg} \overline{\mathbf{N}}_{\mathbf{m n}}$ are those regular at the origin. Note that a bold symbol with one bar represents the column of $(\theta, \varphi, r)$ components of a vector in the spherical coordinate system, while the symbol with double bars represent a matrix.

The T-matrix is now defined by

$$
\left[\begin{array}{l}
p_{m^{\prime} n^{\prime}} \\
q_{m^{\prime} n^{\prime}}
\end{array}\right]=\sum_{n=1}^{\infty} \sum_{m=-n}^{n}\left[\begin{array}{ll}
T_{m^{\prime} n^{\prime} m n}^{11} & T_{m^{\prime} n^{\prime} m n}^{12} \\
T_{m^{\prime} n^{\prime} m n}^{21} & T_{m^{\prime} n^{\prime} m n}^{22}
\end{array}\right]\left[\begin{array}{l}
a_{m n} \\
b_{m n}
\end{array}\right] .
$$

Given the T-matrix, the amplitude scattering matrix and the phase matrix can be obtained. For a particle in a fixed-orientation with the incident light direction aligned with the $z$-axis, we need only to consider the T-matrices with $m= \pm 1$. In this special orientation, for an axially symmetric particle, the amplitude scattering matrix is of the Lorenz-Mie type and given by

$S=\left[\begin{array}{cc}S_{2} & 0 \\ 0 & S_{1}\end{array}\right]$,

where the two diagonal elements are

$$
\begin{aligned}
& S_{2}=\sum_{n=1}^{\infty} \frac{2 n+1}{n(n+1)}\left[a_{n} \tau_{n}(\cos \theta)+b_{n} \pi_{n}(\cos \theta)\right], \\
& S_{1}=\sum_{n=1}^{\infty} \frac{2 n+1}{n(n+1)}\left[a_{n} \pi_{n}(\cos \theta)+b_{n} \tau_{n}(\cos \theta)\right] .
\end{aligned}
$$

In Eqs. (10) and (11), $\theta$ is the scattering angle (defined as the angle between the incidence and scattering directions), and the two coefficients are related to the T-matrix elements:

$$
\begin{aligned}
& a_{n}=-\sum_{n^{\prime}=1}^{\infty} \sqrt{\frac{2 n^{\prime}+1}{2 n+1}} i^{n^{\prime}-n}\left[T_{1 n 1 n^{\prime}}^{21}+T_{1 n 1 n^{\prime}}^{22}\right], \\
& b_{n}=-\sum_{n^{\prime}=1}^{\infty} \sqrt{\frac{2 n^{\prime}+1}{2 n+1}} i^{n^{\prime}-n}\left[T_{1 n 1 n^{\prime}}^{11}+T_{1 n 1 n^{\prime}}^{12}\right] .
\end{aligned}
$$

If the incident light is along the negative $z$-axis direction, we have

$$
\begin{aligned}
& a_{n}=-\sum_{n^{\prime}=1}^{\infty} \sqrt{\frac{2 n^{\prime}+1}{2 n+1}} i^{n^{\prime}-n}(-1)^{n+n^{\prime}}\left[-T_{1 n 1 n^{\prime}}^{21}+T_{1 n 1 n^{\prime}}^{22}\right], \\
& b_{n}=-\sum_{n^{\prime}=1}^{\infty} \sqrt{\frac{2 n^{\prime}+1}{2 n+1}} i^{n^{\prime}-n}(-1)^{n+n^{\prime}}\left[T_{1 n 1 n^{\prime}}^{11}-T_{1 n 1 n^{\prime}}^{12}\right] .
\end{aligned}
$$

For a particle that has mirror symmetry with respect to the $x y$-plane, Eqs. (14) and (15) are the same as Eqs. (12) and (13). In the simple case of a radially symmetric spherical particle, we have

$a_{n}=-T_{1 n 1 n}^{22}, b_{n}=-T_{1 n 1 n}^{11}$.

Note that the normalized phase matrix element $P_{22} / P_{11}$ derived from Eq. (9) is unity, even though the particle is nonspherical. For an axially symmetric particle with either a fixed or random orientation, the procedures necessary to calculate the amplitude scattering matrix and Mueller matrix from the T-matrix are described in detail by Mishchenko et al. [21]. In our study, we employ the analytical orientation average algorithm from Mishchenko et al. [21] and Mishchenko [29] in the computation of the phase matrix of randomly oriented particles, and the fixed-orientation algorithm from Mishchenko et al. [21] and Mishchenko [47] when the direction of incidence is not along the symmetry axis.

\subsection{The invariant imbedding method}

We recapture the basic principle of the IIM developed by Johnson [40] to calculate the T-matrix on the basis of an electromagnetic volume integral equation and summarize the major equations involved in the final computation. An arbitrary scattering volume (homogenous or inhomogeneous) can be viewed as an inhomogeneous sphere (i.e., a portion of the sphere has the dielectric properties of the scattering particle and the reminder is medium/vacuum). In the spherical coordinate system, the particle can be discretized in terms of multiple inhomogeneous spherical layers, as shown in Fig. 1(a). The principle of the IIM is to obtain the T-matrix of a larger sphere of $p$ layers based on the T-matrix of a smaller sphere of $p-1$ layers. The initial T-matrix is zero at the origin of the coordinate system.

To represent the T-matrix in a compact form, a combined index $l$ is defined to represent two indices $m$ and $n$ via $l=n(n+1)+m[48]$. If the series expansions in Eqs. (1) and (2) are truncated to $n_{\max }$, the range of $l$ is $\left[1, l_{\max }\right]$ where $l_{\max }=n_{\max }\left(n_{\max }+2\right)$. The T-matrix is now a $l_{\max } \times l_{\max }$ square 


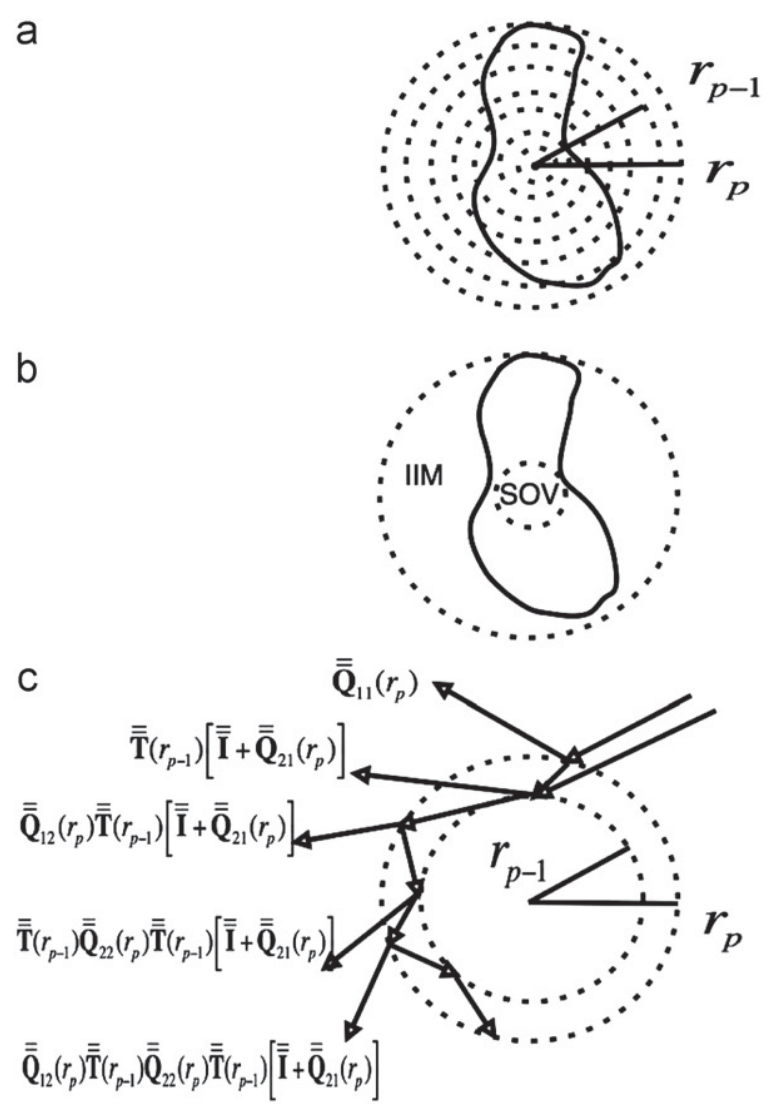

Fig. 1. Schematic geometry of an invariant imbedding approach to compute the T-matrix. (a) Discretize the nonspherical particles in terms of multiple-layered inhomogeneous spheres. (b) A combination of SOV and IIM to compute the T-matrix. (c) The physical interpolation of the IIM equation [17].

supermatrix whose element is a $2 \times 2$ matrix defined in Eq. (8). The fundamental equation to calculate the T-matrix, given by Johnson [40], is

$$
\begin{aligned}
\overline{\overline{\mathbf{T}}}\left(r_{p}\right)= & \overline{\overline{\mathbf{Q}}}_{11}\left(r_{p}\right)+\left[\overline{\overline{\mathbf{I}}}+\overline{\overline{\mathbf{Q}}}_{12}\left(r_{p}\right)\right]\left[\overline{\overline{\mathbf{I}}}-\overline{\overline{\mathbf{T}}}\left(r_{p-1}\right) \overline{\overline{\mathbf{Q}}}_{22}\left(r_{p}\right)\right]^{-1} \\
& \times \overline{\overline{\mathbf{T}}}\left(r_{p-1}\right)\left[\overline{\overline{\mathbf{I}}}+\overline{\mathbf{Q}}_{21}\left(r_{p}\right)\right],
\end{aligned}
$$

where $r_{p}$ is the radius of $p$ th layer,

$$
\overline{\overline{\mathbf{T}}}\left(r_{p}\right)=\left[\begin{array}{ll}
\overline{\overline{\mathbf{T}}}^{11}\left(r_{p}\right) & \overline{\overline{\mathbf{T}}}^{12}\left(r_{p}\right) \\
\overline{\overline{\mathbf{T}}}^{21}\left(r_{p}\right) & \overline{\overline{\mathbf{T}}}^{22}\left(r_{p}\right)
\end{array}\right]
$$

is the T-matrix of a particle composed of $p$ layers, $\overline{\overline{\mathbf{I}}}$ is a $2 l_{\max } \times 2 l_{\max }$ unit matrix, and the supermatrices $\overline{\mathbf{Q}}_{i j}\left(l_{\max } \times\right.$ $\left.l_{\max }\right)$ are defined by

$$
\begin{aligned}
& \overline{\overline{\mathbf{Q}}}_{11}\left(r_{p}\right)=i k \overline{\overline{\mathbf{T}}}\left(r_{p}\right) \overline{\overline{\mathbf{Q}}}\left(r_{p}\right) \overline{\overline{\mathbf{J}}}\left(r_{p}\right), \\
& \overline{\overline{\mathbf{Q}}}_{12}\left(r_{p}\right)=i k \overline{\overline{\mathbf{T}}}\left(r_{p}\right) \overline{\overline{\mathbf{Q}}}\left(r_{p}\right) \overline{\overline{\mathbf{H}}}\left(r_{p}\right), \\
& \overline{\overline{\mathbf{Q}}_{2,1}}\left(r_{p}\right)=i k \overline{\overline{\mathbf{H}^{T}}}\left(r_{p}\right) \overline{\overline{\mathbf{Q}}}\left(r_{p}\right) \overline{\overline{\mathbf{J}}}\left(r_{p}\right), \\
& \overline{\overline{\mathbf{Q}}}_{2,2}\left(r_{p}\right)=i k \overline{\overline{\mathbf{H}^{T}}}\left(r_{p}\right) \overline{\overline{\mathbf{Q}}}\left(r_{p}\right) \overline{\overline{\mathbf{H}}}\left(r_{p}\right),
\end{aligned}
$$

where $\overline{\overline{\mathbf{J}}}\left(r_{p}\right)$ and $\overline{\overline{\mathbf{H}}}\left(r_{p}\right)$ are $l_{\max } \times l_{\text {max }}$ diagonal supermatrices with each diagonal element defined by Eq. (6). $\overline{\mathbf{Q}}\left(r_{p}\right)$ and the relevant quantities are given by

$$
\begin{aligned}
& \overline{\overline{\mathbf{Q}}}\left(r_{p}\right)=w_{p} \frac{\overline{\overline{\mathbf{U}}}\left(r_{p}\right)}{\left[\overline{\overline{\mathbf{I}}}-w_{p} \overline{\overline{\mathbf{U}}}\left(r_{p}\right) \overline{\bar{g}}\left(r_{p}, r_{p}\right)\right]}, \\
& \overline{\overline{\mathbf{U}}}_{l(m n) l^{\prime}\left(m^{\prime} n^{\prime}\right)}\left(r_{n}\right)=k^{2} r_{n}^{2} \int d \Omega \overline{\overline{\mathbf{Y}}}_{l(m n)}^{T *}(\theta, \phi) \\
& \times\left[\tilde{m}(\vec{r})^{2}-1\right] \overline{\overline{\mathbf{Z}}}(\vec{r}) \overline{\overline{\mathbf{Y}}}_{l^{\prime}\left(m^{\prime} n^{\prime}\right)}(\theta, \phi), \\
& \overline{\overline{\mathbf{Z}}}(\vec{r})=\left[\begin{array}{lll}
1 / \tilde{m}^{2} & 0 & 0 \\
0 & 1 & 0 \\
0 & 0 & 1
\end{array}\right] \\
& \overline{\bar{g}}_{n}\left(r, r^{\prime}\right)=\left\{\begin{array}{l}
i k \overline{\overline{\mathbf{H}}}_{n}(r) \overline{\overline{\mathbf{J}}}_{n}^{T}\left(r^{\prime}\right) ; \quad r>r^{\prime} \\
\frac{i k}{2}\left[\overline{\overline{\mathbf{H}}}_{n}(r) \overline{\mathbf{J}}_{n}^{T}\left(r^{\prime}\right)+\overline{\overline{\mathbf{J}}}_{n}(r) \overline{\overline{\mathbf{H}}}_{n}^{T}\left(r^{\prime}\right)\right] ; \quad r=r^{\prime} . \\
i k \overline{\overline{\mathbf{J}}}_{n}(r) \overline{\overline{\mathbf{H}}}_{n}^{T}{ }_{n}\left(r^{\prime}\right) ; \quad r<r^{\prime}
\end{array}\right.
\end{aligned}
$$

In Eq. (22), $\overline{\overline{\mathbf{U}}}$ is a $l_{\max } \times l_{\max }$ supermatrix with each element defined in Eq. (23), $\mathrm{g}$ is a $l_{\max } \times l_{\max }$ diagonal supermatrix with each element defined in Eq. (25), and $w_{n}$ is the weight at each discrete radius. In Eqs. (23) and (24), $\tilde{m}$ is the refractive index. Eqs. (17)-(25) are similar to those in Section E in Johnson [40]; however, they differ because our formalism is based on exponential $\varphi$ dependence and Johnson employed even and odd functions. An explicit derivation of Eq. (17) based on the exponential $\varphi$ dependence is given in Appendix.

The direct application of Eq. (17) to numerical computation is inefficient. To overcome the computational inefficiency, the SOV method can be employed to calculate the T-matrix of the sphere centered at the origin and inscribed within the scattering particle to the maximum extent. The IIM is applied in the volume between the circumscribed sphere and the inscribed sphere centered in the coordinate systems as shown in Fig. 1(b). The Lorenz-Mie coefficients are related to the T-matrix elements through Eq. (16); therefore, Eqs. (16)-(25) provide a closed set of mathematical equations to solve Maxwell's equations.

Johnson [40] did not discuss the physical interpolation of Eq. (17), which is shown in Fig. 1(c). The contribution of the surface $\left(r_{p}\right)$ independent to the scattering is given by $\overline{\mathbf{Q}}_{11}$, the reflection of the incident source and the source from the surface $r_{p}$ at the surface $r_{p-1}$ is given by $\overline{\overline{\mathbf{T}}}\left(r_{p-1}\right)\left[\overline{\overline{\mathbf{I}}}+\overline{\overline{\mathbf{Q}}}_{2,1}\left(r_{p}\right)\right]$, and the contribution of the source from the surface $r_{p-1}$ after interaction with the surface $r_{p}$ is $\overline{\mathbf{Q}}_{12}\left(r_{p}\right) \overline{\overline{\mathbf{T}}}\left(r_{p-1}\right)\left[\overline{\overline{\mathbf{I}}}+\overline{\mathbf{Q}}_{2,1}\left(r_{p}\right)\right]$. Multiple interactions between two surfaces are taken into account through matrix inversion:

$\left[\overline{\overline{\mathbf{I}}}-\overline{\overline{\mathbf{T}}}\left(r_{p-1}\right) \overline{\overline{\mathbf{Q}}}_{2,2}\left(r_{p}\right)\right]^{-1}=\sum_{k=0}^{\infty}\left[\overline{\overline{\mathbf{T}}}\left(r_{p-1}\right) \overline{\overline{\mathbf{Q}}}_{2,2}\left(r_{p}\right)\right]^{k}$.

Note that the arrows in Fig. 1(c) explain the interaction relation involved in Eq. (17) and should not be construed as rays of light in geometric optics.

The only shape-and-refractive-index-dependent part of the computational program is the U-matrix defined in 
Eq. (23), which can be written by the following explicit expression,

Based on Eqs. (30) and (31),

$$
T_{m n m n^{\prime}}^{11}=T_{m n m n^{\prime}}^{22}=0 \text {, when }(-1)^{n+n^{\prime}}=-1 \text {, }
$$

$$
\begin{gathered}
\left.\overline{\overline{\mathbf{U}}}_{m n m^{\prime} n^{\prime}}=k^{2} r^{2}(-1)^{m+m^{\prime}}\left[\frac{2 n+1}{4 \pi n(n+1)}\right]^{1 / 2}\left[\frac{2 n^{\prime}+1}{4 \pi n^{\prime}\left(n^{\prime}+1\right)}\right]^{1 / 2} \int_{0}^{2 \pi} d \phi \int_{0}^{\pi} d \theta \sin \theta \exp \left[-i\left(m-m^{\prime}\right) \phi\right][\varepsilon(r, \theta, \phi)-1)\right] \\
{\left[\begin{array}{ccc}
\pi_{m n}(\theta) \pi_{m^{\prime} n^{\prime}}(\theta)+\tau_{m n}(\theta) \tau_{m^{\prime} n^{\prime}}(\theta) & -i\left[\pi_{m n}(\theta) \tau_{m^{\prime} n^{\prime}}(\theta)+\tau_{m n}(\theta) \pi_{m^{\prime} n^{\prime}}(\theta)\right] & 0 \\
i\left[\pi_{m n}(\theta) \tau_{m^{\prime} n^{\prime}}(\theta)+\tau_{m n}(\theta) \pi_{m^{\prime} n^{\prime}}(\theta)\right] & \pi_{m n}(\theta) \pi_{m^{\prime} n^{\prime}}(\theta)+\tau_{m n}(\theta) \tau_{m^{\prime} n^{\prime}}(\theta) & 0 \\
0 & 0 & \sqrt{n n^{\prime}(n+1)\left(n^{\prime}+1\right)} d_{0 m}^{n}(\theta) d_{0 m^{\prime}}^{n \prime}(\theta) / m^{2}(r, \theta, \phi)
\end{array}\right] .}
\end{gathered}
$$

In comparison with the EBCM, modifying the composition of the scattering particle without modifying the system of equations is simpler and significantly reduces the complexity of numerical implementation.

The symmetry of the T-matrix can be explicitly understood from the U-matrix. If the particle is of axially rotational symmetry, after integration in terms of $\varphi$, Eq. (27) simplifies to

$$
\begin{aligned}
& \left.\overline{\overline{\mathbf{U}}}_{m n m^{\prime} n^{\prime}}=\delta_{m m^{\prime}} k^{2} r^{2} \frac{1}{2}\left[\frac{2 n+1}{n(n+1)}\right]^{1 / 2}\left[\frac{2 n^{\prime}+1}{n^{\prime}\left(n^{\prime}+1\right)}\right]^{1 / 2} \int_{0}^{\pi} d \theta \sin \theta[\varepsilon(r, \theta)-1)\right] \\
& {\left[\begin{array}{ccc}
\pi_{m n}(\theta) \pi_{m n^{\prime}}(\theta)+\tau_{m n}(\theta) \tau_{m n^{\prime}}(\theta) & -i\left[\pi_{m n}(\theta) \tau_{m n^{\prime}}(\theta)+\tau_{m n}(\theta) \pi_{m n^{\prime}}(\theta)\right] & 0 \\
i\left[\pi_{m n}(\theta) \tau_{m n^{\prime}}(\theta)+\tau_{m n}(\theta) \pi_{m n^{\prime}}(\theta)\right] & \pi_{m n}(\theta) \pi_{m n^{\prime}}(\theta)+\tau_{m n}(\theta) \tau_{m n^{\prime}}(\theta) & 0 \\
0 & 0 & \sqrt{n n^{\prime}(n+1)\left(n^{\prime}+1\right)} d_{0 m}^{n}(\theta) d_{0 m}^{d^{\prime \prime}}(\theta) \\
\varepsilon(r, \theta)
\end{array}\right] .}
\end{aligned}
$$

Note that $[\varepsilon(r, \theta)-1)]$ is a step function in terms of $\theta$ and is zero when $\theta$ is outside the particle. If Eq. (28) is valid at each discrete radius, the T-matrix must have the following property

$\overline{\overline{\mathbf{T}}}_{m n m^{\prime} n^{\prime}}=\delta_{m m^{\prime}} \overline{\overline{\mathbf{T}}}_{m n m n^{\prime}}$,

where we denote

$\overline{\overline{\mathbf{T}}}_{m n m^{\prime} n^{\prime}}=\left[\begin{array}{ll}T_{m n m^{\prime} n^{\prime}}^{11} & T_{m n m^{\prime} n^{\prime}}^{12} \\ T_{m n m^{\prime} n^{\prime}}^{21} & T_{m n m^{\prime} n^{\prime}}^{22}\end{array}\right]$.

If the axially symmetric particles have mirror symmetry with respect to the $x y$-plane, the $\mathrm{U}$-matrix further simplifies to
$T_{m n m n^{\prime}}^{12}=T_{m n m n^{\prime}}^{21}=0$, when $(-1)^{n+n^{\prime}}=1$

In the case of a homogenous sphere, employing the orthogonal relationships of

$\int_{0}^{\pi} d \theta \sin \theta\left[\pi_{m n}(\theta) \tau_{m n^{\prime}}(\theta)+\tau_{m n}(\theta) \pi_{m n^{\prime}}(\theta)\right]=0$, $\int_{0}^{\pi} d \theta \sin \theta\left[\pi_{m n}(\theta) \pi_{m n^{\prime}}(\theta)+\tau_{m n}(\theta) \tau_{m n^{\prime}}(\theta)\right]=\frac{2 n(n+1)}{2 n+1} \delta_{n n^{\prime}}$,

$\int_{0}^{\pi} d \theta \sin \theta d_{0 m}^{n}(\theta) d_{0 m}^{n \prime}(\theta)=\frac{2}{2 n+1} \delta_{n n^{\prime}}$,

gives the $\mathrm{U}$-matrix in a diagonal form:

$\overline{\overline{\mathbf{U}}}_{m n m^{\prime} n^{\prime}}=k^{2} r^{2}(\varepsilon-1) \delta_{m m^{\prime}} \delta_{n n^{\prime}}\left[\begin{array}{ccc}1 & 0 & 0 \\ 0 & 1 & 0 \\ 0 & 0 & 1 / \varepsilon\end{array}\right]$.

Realizing the T-matrix symmetry in the case of an axially symmetric nonspherical particle, Eq. (17) can be separated into $n_{\max }+1$ equations according to the index $m$ :

$$
\begin{aligned}
& \left.\overline{\mathbf{U}}_{m n m^{\prime} n^{\prime}}=\delta_{m m^{\prime}} k^{2} r^{2} \frac{1}{2}\left[\frac{2 n+1}{n(n+1)}\right]^{1 / 2}\left[\frac{2 n^{\prime}+1}{n^{\prime}\left(n^{\prime}+1\right)}\right]^{1 / 2} \int_{0}^{\pi / 2} d \theta \sin \theta[\varepsilon(r, \theta)-1)\right] \\
& {\left[\begin{array}{ccc}
c_{n n^{\prime}}\left[\pi_{m n}(\theta) \pi_{m n^{\prime}}(\theta)+\tau_{m n}(\theta) \tau_{m n^{\prime}}(\theta)\right]^{\prime} & -i \tilde{c}_{n n^{\prime}}\left[\pi_{m n}(\theta) \tau_{m n^{\prime}}(\theta)+\tau_{m n}(\theta) \pi_{m n^{\prime}}(\theta)\right] & 0 \\
i \tilde{c}_{n n^{\prime}}\left[\pi_{m n}(\theta) \tau_{m n^{\prime}}(\theta)+\tau_{m n}(\theta) \pi_{m n^{\prime}}(\theta)\right] & c_{n n^{\prime}}\left[\pi_{m n}(\theta) \pi_{m n^{\prime}}(\theta)+\tau_{m n}(\theta) \tau_{m n^{\prime}}(\theta)\right] & 0 \\
0 & 0 & \frac{c_{n n^{\prime}} \sqrt{n n^{\prime}(n+1)\left(n^{\prime}+1\right)} d_{0 m}^{n}(\theta) d_{0 m}^{n \prime}(\theta)}{\varepsilon(r, \theta)}
\end{array}\right]}
\end{aligned}
$$

where

$c_{n n^{\prime}}=\left[1+(-1)^{n+n^{\prime}}\right], \tilde{c}_{n n^{\prime}}=\left[1+(-1)^{n+n^{\prime}+1}\right]$,

$$
\begin{aligned}
& \overline{\overline{\mathbf{T}}}_{m n m n^{\prime}}\left(r_{p}\right)=\overline{\overline{\mathbf{Q}}}_{11}^{m}\left(r_{p}\right)+\left[\overline{\overline{\mathbf{I}}}+\overline{\overline{\mathbf{Q}}}_{12}^{m}\left(r_{p}\right)\right]\left[\overline{\overline{\mathbf{I}}}-\overline{\overline{\mathbf{T}}}_{m n m n^{\prime}}\left(r_{p-1}\right)\right. \\
& \left.\times \overline{\overline{\mathbf{Q}}}^{m}{ }_{22}\left(r_{p}\right)\right]^{-1} \overline{\overline{\mathbf{T}}}_{m n m n^{\prime}}\left(r_{p-1}\right)\left[\overline{\overline{\mathbf{I}}}+\overline{\overline{\mathbf{Q}}}_{21}^{m}\left(r_{p}\right)\right] .
\end{aligned}
$$


For each $m$, the size of the T-matrix is $2\left(n_{\max }-m+1\right) \times 2\left(n_{\max }-m+1\right)$. If the incident direction is along the axis, $m=1$ is sufficient to obtain the solution. For an arbitrarily incident direction (particle orientation) or randomly oriented particle, $m$ ranges from 0 to $n_{\max }$. In the case of a sphere, Eq. (17) becomes $n_{\max }$ separate equations in which the T-matrix is a $2 \times 2$ diagonal matrix.

\subsection{Numerical implementations}

The truncation number and the number of spherical layers to discretize the particle are two fundamental parameters to guarantee the convergence of the T-matrix. In the numerical calculations, the two computational parameters are increased such that the solutions converge with acceptable precision. At different spherical surfaces, the T-matrix is truncated to a different number (i.e., the remaining elements in the T-matrix not involved in the computation are assumed to be zero) to overcome the overflow problem and increase the computational speed. The Gaussian quadrature is employed for the computation of the U-matrix (28), and the number of division points is increased so that the numerical error is smaller than a prescribed value (e.g., $10^{-10}$ ).

Clebsch-Gordan coefficients are indispensable in the analytical orientation average algorithm. The twodirectional recursive algorithm [21] is employed for fast computation. The center value of the index $n^{\prime}$ of $C^{n^{\prime} 1}{ }_{n m n 1,1-m}$ in Eq. (5.122), given in Appendix D in [21], is problematic when $n^{\prime}$ is larger than $\sim 150$. Instead, a computational procedure is implemented to search the center of the classical region. After the correction, the random-orientation averaging algorithm is tested for a sphere with a particle size parameter larger than 500 and gives the same results as its counterpart computed from the Lorenz-Mie theory.

Computer memory requirements increase dramatically with respect to the particle size parameter. To reduce the memory burden that limits the largest applicable size parameter, we: (a) store non-zero T-matrix elements based on Eqs. (32) and (33) when the particle has a mirror symmetry with respect to the $x y$-plane; (b) reduce the three-dimensional $D$ arrays in Eqs. (5.126)-(5.130) of Ref. [21] to two dimensional arrays by switching the loops in terms of the indices $s$ and $n$; (c) store non-zero values of the $B$-array $B_{m n n 1}^{j}$ defined in Eq. (5.122) of Ref. [21]; and, (d) store the T-matrix elements and $B$-array in singleprecision variables. $B_{m n n_{1}}^{j}$ is proven to be zero when $n_{1}$ is odd because of the symmetry of indices inherent in Clebsch-Gordan coefficients and the T-matrix. After the T-matrix elements for each index $m$ are obtained in double precision, the single-precision and doubleprecision array to store T-matrix elements and $B$-array are found to give the same results within several decimal places. The final memory demand for a particle size parameter of 500 , defined in terms of the radius of a circumscribed sphere, is less than 3 GB.

To apply the IIM T-matrix method to axially symmetric particles with large size parameters, we have parallelized the Fortran 90 program based on the Message Passing Interface (MPI) technology. The calculations of sub
T-matrices are distributed to different processors according to the index $m$. As the index $m$ increases, the size of the T-matrix decreases. As a result, the computation time significantly decreases for large values of $m$. When the incident light direction is aligned with the symmetry axis, the algorithm does not need to be parallelized, because solving the equation for $m=1$ is sufficient to obtain the scattering solution.

Furthermore, reciprocity conditions and other relationships [49] are employed to check the T-matrix and expansion coefficients of the phase matrix. All the equalities are in full agreement and the expansion coefficients of the phase matrix passed the check of the subroutine HOVENR in the Mishchenko's EBCM program. For nonabsorbing particles, the single-scattering albedo is found to be slightly greater than unity; however, the value is accurate to a significant number of places.

\section{Results and discussion}

\subsection{Testing against EBCM: spheroids and cylinders}

We apply the IIM + SOV method to homogenous spheroids and cylinders whose optical properties have been extensively studied by using the standard EBCM. A commonly used EBCM code for spheroids and cylinders written by Mishchenko and Travis [50] is publicly available at http://www.giss.nasa.gov/ crmim. The maximum size parameter handled by the EBCM decreases as the aspect ratio becomes extreme (i.e., very long or flat spheroids). In the extended-precision calculations, the maximum size parameter is larger than in the doubleprecision calculations. We have tested the IIM+SOV program (double-precision variables are used in the calculation of the T-matrix) in various cases calculated by the EBCM and excellent agreement between the two methods has been obtained.
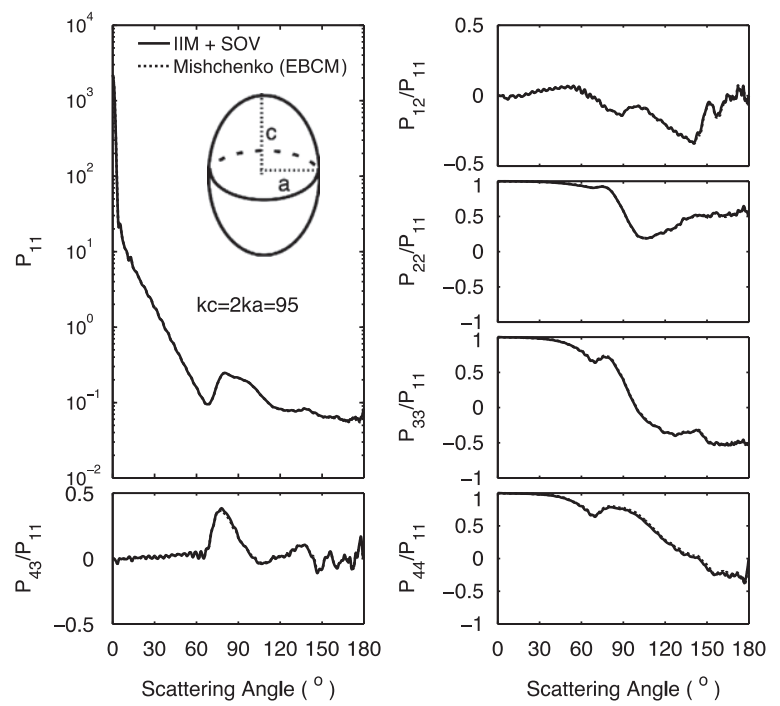

Fig. 2. Comparison of phase matrix elements simulated from the EBCM and the IIM+SOV for randomly oriented spheroids. 
Table 1

The values of six nonzero phase matrix elements of randomly oriented prolate spheroids at 9 scattering angles simulated from the EBCM and the IIM+SOV. The "Diff" rows in the table cells indicate the differences between the results computed from the two methods.

\begin{tabular}{|c|c|c|c|c|c|c|}
\hline$\theta\left(^{\circ}\right)$ & $P_{11}$ & $P_{22}$ & $P_{33}$ & $P_{44}$ & $P_{12}$ & $P_{34}$ \\
\hline \multicolumn{7}{|l|}{0} \\
\hline EBCM & 16.3398 & 16.2871 & 16.2871 & 16.2345 & 0.0000 & 0.0000 \\
\hline SOV+IIM & 16.3415 & 16.2919 & 16.2919 & 16.2423 & 0.0000 & 0.0000 \\
\hline Diff & -0.0017 & -0.0048 & -0.0048 & -0.0078 & 0.0000 & 0.0000 \\
\hline \multicolumn{7}{|l|}{30} \\
\hline EBCM & 4.9774 & 4.9449 & 4.9043 & 4.8938 & -0.2142 & 0.5491 \\
\hline SOV+IIM & 4.9772 & 4.9452 & 4.9045 & 4.8943 & -0.2074 & 0.5525 \\
\hline Diff & 0.0002 & -0.0003 & -0.0002 & -0.0005 & -0.0068 & -0.0034 \\
\hline \multicolumn{7}{|l|}{60} \\
\hline EBCM & 0.2987 & 0.2835 & 0.2156 & 0.2257 & 0.0976 & 0.0596 \\
\hline SOV+IIM & 0.2989 & 0.2836 & 0.2151 & 0.2252 & 0.0958 & 0.0590 \\
\hline Diff & -0.0002 & -0.0001 & 0.0005 & 0.0005 & 0.0018 & 0.0006 \\
\hline \multicolumn{7}{|l|}{90} \\
\hline EBCM & 0.1459 & 0.1220 & 0.0755 & 0.0970 & -0.0471 & -0.0340 \\
\hline SOV+IIM & 0.1457 & 0.1217 & 0.0759 & 0.0972 & -0.0470 & -0.0340 \\
\hline Diff & 0.0002 & 0.0003 & -0.0004 & -0.0002 & -0.0001 & 0.0000 \\
\hline \multicolumn{7}{|l|}{120} \\
\hline ЕВСМ & 0.0621 & 0.0356 & 0.0027 & 0.0272 & -0.0094 & 0.0103 \\
\hline SOV+IIM & 0.0620 & 0.0355 & 0.0025 & 0.0269 & -0.0094 & 0.0103 \\
\hline Diff & 0.0001 & 0.0001 & 0.0002 & 0.0003 & 0.0000 & 0.0000 \\
\hline \multicolumn{7}{|l|}{150} \\
\hline EBCM & 0.0326 & 0.0244 & -0.0197 & -0.0132 & 0.0024 & -0.0058 \\
\hline SOV+IIM & 0.0328 & 0.0244 & -0.0198 & -0.0132 & 0.0024 & -0.0058 \\
\hline Diff & -0.0002 & 0.0000 & 0.0001 & 0.0000 & 0.0000 & 0.0000 \\
\hline \multicolumn{7}{|l|}{180} \\
\hline EBCM & 0.0585 & 0.0329 & -0.0329 & -0.0074 & 0.0000 & 0.0000 \\
\hline SOV + IIM & 0.0583 & 0.0327 & -0.0327 & -0.0071 & 0.0000 & 0.0000 \\
\hline Diff & 0.0002 & -0.0002 & -0.0002 & -0.0003 & 0.0000 & 0.0000 \\
\hline
\end{tabular}

Table 2

The extinction efficiency, the scattering efficiency, the absorption efficiency, the single-scattering albedo, and the asymmetry factor simulated from the EBCM and the IIM+SOV. The "Diff" row in the table cells indicates the differences between the results computed from the two methods.

\begin{tabular}{llllll}
\hline & Qext & Qsca & Qabs & $\omega$ & $\langle\cos \theta\rangle$ \\
\hline EBCM & 3.2854 & 2.2903 & 0.9951 & 0.6971 & 0.8165 \\
SOV + IIM & 3.2850 & 2.2901 & 0.9949 & 0.6971 & 0.8165 \\
Diff & 0.0004 & 0.0002 & 0.0002 & 0.0000 & 0.0000 \\
\hline
\end{tabular}

Table 1 presents the comparison of the six nonzero phase matrix elements of a prolate spheroid in random orientation computed from the IIM+SOV (the second row for each scattering angle) and the benchmark results (the first row for each scattering angle) computed from the EBCM [29]. The ratio of semi-major axis (b) over semi-minor axis (a) is 2, the size parameter $(\mathrm{kb})$ is 5.5 , and the refractive index is $1.5+0.1 i$. The IIM + SOV can reproduce up to four decimal places depending on the scattering angle. In the computation, a Romberg integration technique is employed to improve the

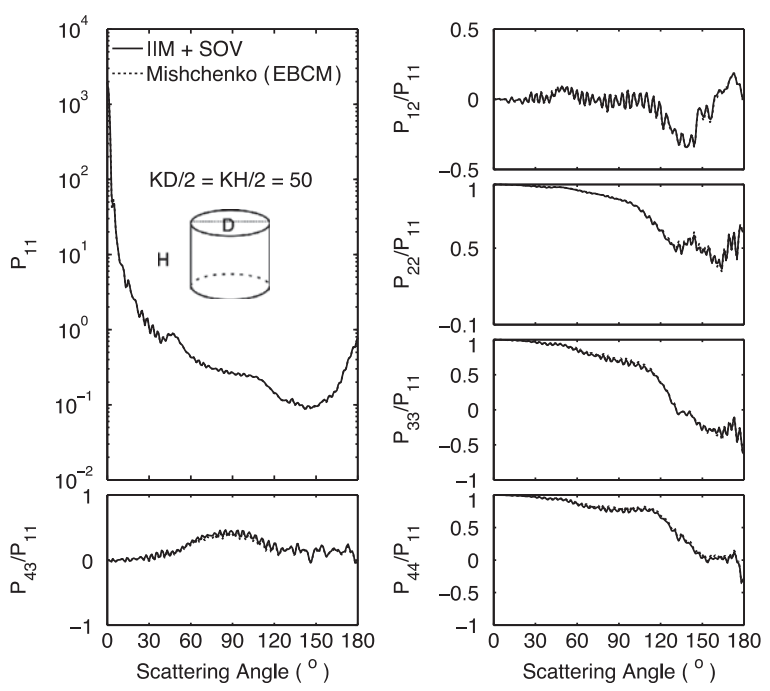

Fig. 3. Comparison of phase matrix elements simulated from the EBCM and the IIM+SOV for randomly oriented compact cylinders. 
Table 3

Similar to Table 1, but for randomly oriented cylinders.

\begin{tabular}{|c|c|c|c|c|c|c|}
\hline$\theta\left(^{\circ}\right)$ & $P_{11}$ & $P_{22}$ & $P_{33}$ & $P_{44}$ & $P_{12}$ & $P_{34}$ \\
\hline \multicolumn{7}{|l|}{0} \\
\hline EBCM & 9.8167 & 9.7796 & 9.7796 & 9.7426 & 0.0000 & 0.0000 \\
\hline $\mathrm{SOV}+\mathrm{IIM}$ & 9.8176 & 9.7817 & 9.7817 & 9.7458 & 0.0000 & 0.0000 \\
\hline Diff & -0.0009 & -0.0021 & -0.0021 & -0.0032 & 0.0000 & 0.0000 \\
\hline \multicolumn{7}{|l|}{30} \\
\hline EBCM & 4.5720 & 4.5226 & 4.5064 & 4.5045 & -0.2996 & 0.2008 \\
\hline $\mathrm{SOV}+\mathrm{IIM}$ & 4.5732 & 4.5242 & 4.5085 & 4.5068 & -0.2932 & 0.2010 \\
\hline Diff & -0.0012 & -0.0016 & -0.0021 & -0.0023 & -0.0064 & -0.0002 \\
\hline \multicolumn{7}{|l|}{60} \\
\hline EBCM & 0.8046 & 0.7415 & 0.7084 & 0.7536 & -0.0769 & 0.1585 \\
\hline SOV+IIM & 0.8043 & 0.7411 & 0.7075 & 0.7532 & -0.0765 & 0.1581 \\
\hline Diff & 0.0003 & 0.0004 & 0.0009 & 0.0004 & -0.0004 & 0.0004 \\
\hline \multicolumn{7}{|l|}{90} \\
\hline EBCM & 0.1972 & 0.1442 & 0.1151 & 0.1595 & 0.0367 & -0.0007 \\
\hline SOV+IIM & 0.1973 & 0.1440 & 0.1148 & 0.1594 & 0.0363 & -0.0009 \\
\hline Diff & -0.0001 & 0.0002 & 0.0003 & 0.0001 & 0.0004 & -0.0002 \\
\hline \multicolumn{7}{|l|}{120} \\
\hline EBCM & 0.1080 & 0.0840 & -0.0193 & -0.0006 & -0.0106 & -0.0479 \\
\hline SOV+IIM & 0.1077 & 0.0835 & -0.0190 & -0.0002 & -0.0108 & -0.0481 \\
\hline Diff & 0.0003 & 0.0005 & -0.0003 & -0.0004 & 0.0002 & 0.0002 \\
\hline \multicolumn{7}{|l|}{150} \\
\hline EBCM & 0.1226 & 0.1061 & -0.0964 & -0.0819 & -0.0042 & -0.0146 \\
\hline $\mathrm{SOV}+\mathrm{IIM}$ & 0.1223 & 0.1057 & -0.0960 & -0.0815 & -0.0043 & -0.0145 \\
\hline Diff & 0.0003 & 0.0004 & -0.0004 & -0.0004 & 0.0001 & -0.0001 \\
\hline \multicolumn{7}{|l|}{180} \\
\hline EBCM & 0.1731 & 0.1261 & -0.1261 & -0.0790 & 0.0000 & 0.0000 \\
\hline $\mathrm{SOV}+\mathrm{IIM}$ & 0.1730 & 0.1259 & -0.1259 & -0.0788 & 0.0000 & 0.0000 \\
\hline Diff & 0.0001 & 0.0002 & -0.0002 & -0.0002 & 0.0000 & 0.0000 \\
\hline
\end{tabular}

Table 4

Similar to Table 2, but for randomly oriented cylinders.

\begin{tabular}{lllllr}
\hline & Qext & Qsca & Qabs & $\omega$ & $\langle\cos \theta\rangle$ \\
\hline EBCM & 2.5322 & 2.4471 & 0.0851 & 0.9664 & 0.7090 \\
SOV + IIM & 2.5313 & 2.4462 & 0.0851 & 0.9664 & 0.7092 \\
Diff & 0.0009 & 0.0009 & 0.0000 & 0.0000 & -0.0002 \\
\hline
\end{tabular}

numerical accuracy of the T-matrix until the decimal points in the table will not change by increasing the number of spherical layers to discretize the spheroid. The truncation number is increased in order for the final results to converge. Although some decimal points vary between the results computed from the two different methods, the forward and backscattering phase matrix computed from the two methods satisfies the inherent relations: $-P_{11}+P_{22}+P_{33}-P_{44}=0$ at the forward scattering angle and $P_{11}-P_{22}+P_{33}-P_{44}=0$ at the backscattering angle [21]. Table 2 shows a comparison between the extinction efficiency, the scattering efficiency, the absorption efficiency, the single-scattering albedo, and the asymmetry factor computed by the two methods. The results agree up to four decimal places.

Fig. 2 shows a comparison between the phase matrix of a large randomly oriented prolate spheroid computed with the EBCM and with a combination of IIM and SOV. The ratio of the semi-major to the semi-minor axis is 2 . The size parameter, defined in terms of the semi-major axis, is 95. The refractive index is chosen to be 1.311 , which was used to test the computational capabilities of the EBCM (e.g., $[34,50]$ ) and is used as a reference in the present study. The comparison exhibits virtually the same results. The computational time is approximately $22 \mathrm{~min}$ using $32(2.8 \mathrm{GHz})$ processors for the IIM+SOV method and $13 \mathrm{~min}$ using a single $(2.8 \mathrm{GHz})$ processor for the extended-precision EBCM. Thus, using the EBCM is highly recommended within its applicable size parameter regime due to its significantly shorter processing time.

Numerical simulations with cylinders have also been carried out, similar to those for spheroids. Tables 3 and 4 show the optical properties of a randomly oriented circular cylinder with an equal-surface-area-sphere size parameter of 3 , a diameter-to-height ration of 0.5 , and a refractive index of $1.53+0.008$ i [51]. Similar accuracy to that illustrated in Tables 1 and 2 is obtained. Fig. 3 shows the comparison of six independent non-zero phase matrix elements for a large randomly oriented cylinder simulated from the EBCM and the IIM+SOV for a size parameter of $\{k D / 2=50, k H / 2=50\}$, where $D$ is the diameter and $H$ is the height. As demonstrated 
in Fig. 3, an overall agreement is achieved but small differences are noticeable in the $P_{43}$ element. A halo near $46^{\circ}$, commensurate with geometric optics, is evident at this size parameter. A discussion of the particle size necessary to produce halos has been reported where the phase function of a cylindrical particle was computed with the EBCM for size parameters of $40,80,120$, and 180 , with the size parameter defined in terms of $k D / 2$ [52].

\subsection{Comparison with the improved geometric optics method: large spheroids and cylinders}

Fig. 4 shows the application of the IIM + SOV method to a size parameter of 300 , i.e., where no other rigorous or
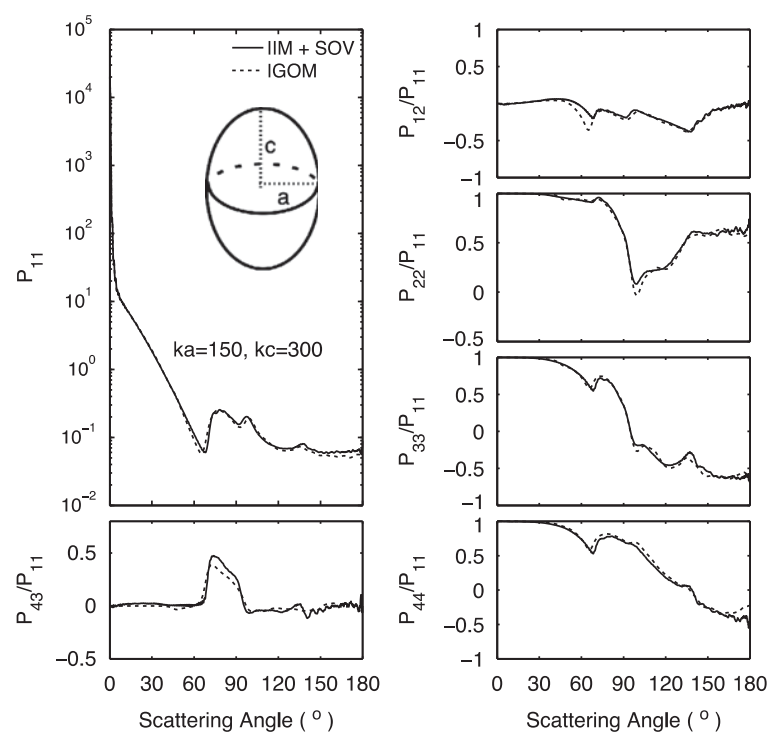

Fig. 4. Comparison of phase matrix elements simulated from the $\mathrm{IIM}+\mathrm{SOV}$ and the IGOM for randomly oriented spheroids.
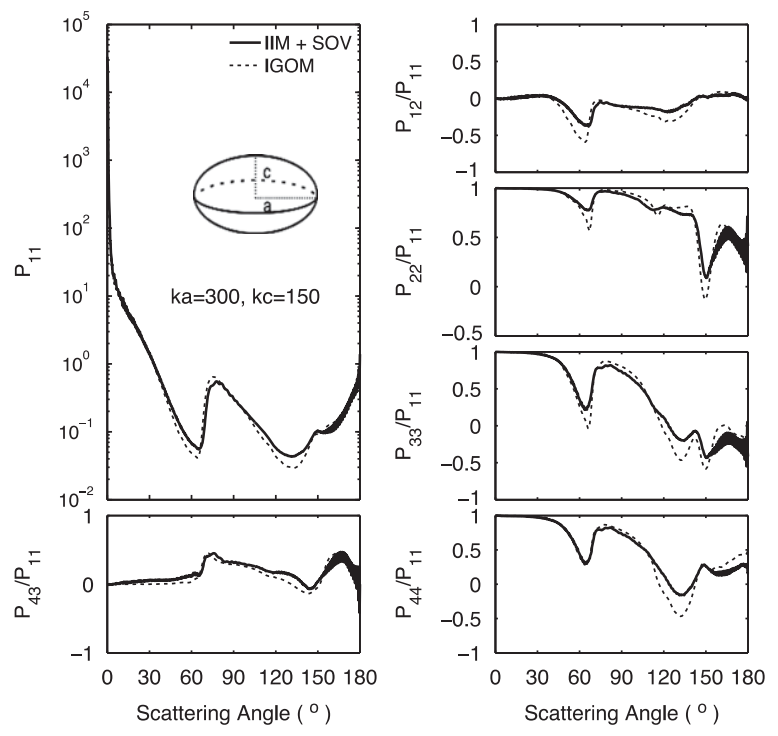

Fig. 5. Similar to Fig. 4, but for oblate spheroids. numerical methods are currently applicable. The simulated results are compared with the approximate geometric-optics results computed with the Improved Geometric-Optics Method (IGOM) [14]. As demonstrated in Fig. 4, the geometric-optics results are similar to those computed from the IIM+SOV. Note that the interference among rays is neglected in the IGOM calculations and explains the missing oscillations in the IGOM results. According to Mishchenko and Travis [50], for the extended-precision EBCM, the maximum convergent size parameter of a prolate spheroid with an aspect ratio of 2 is 112 for the semi-major axis. Similar to Fig. 4, Fig. 5 shows an oblate spheroid case. In Ref. [34], the EBCM was applied to large oblate spheroids with semi-major axis size parameters up to 102.3216 , which has a surfaceequivalent-sphere size parameter of 85 . The present size
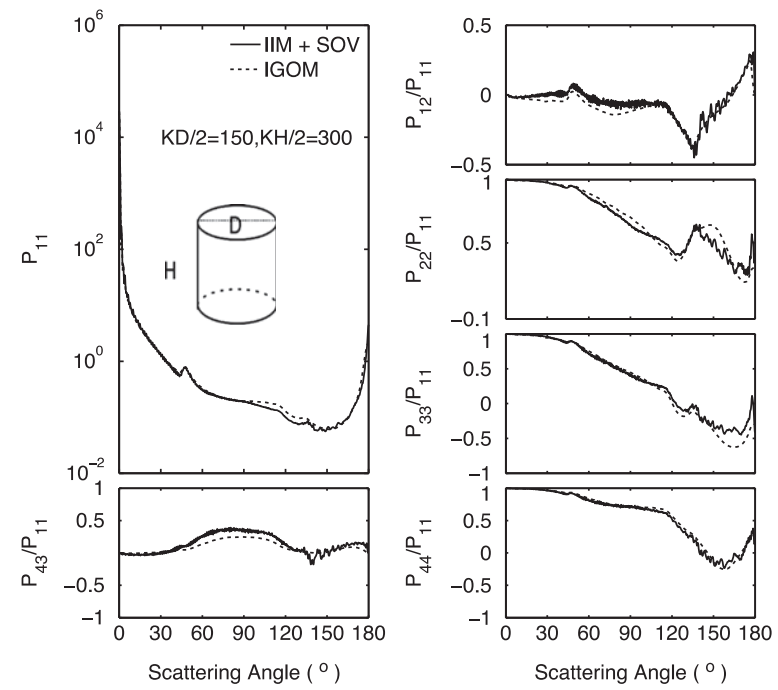

Fig. 6. Comparison of phase matrix elements simulated from the IIM + SOV and the IGOM for randomly oriented prolate cylinders.
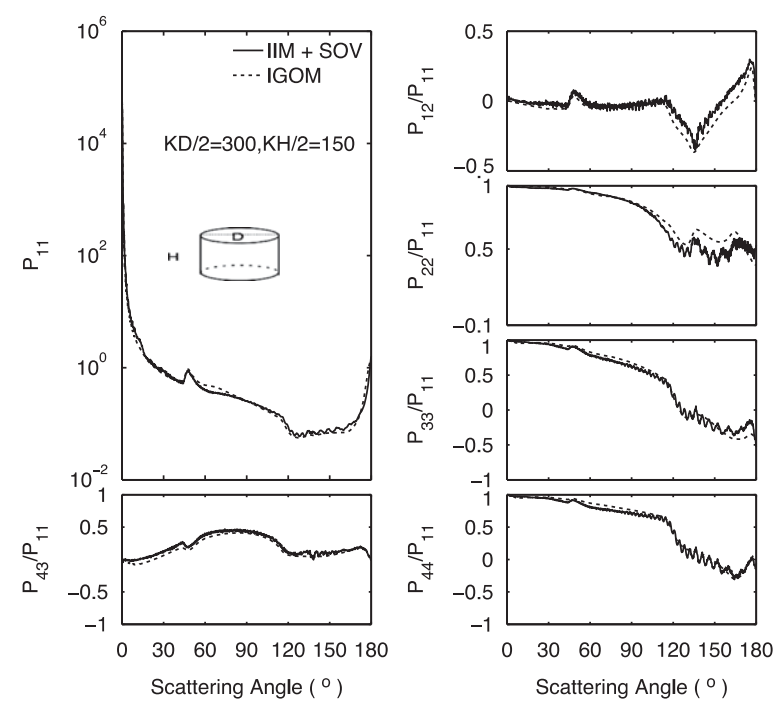

Fig. 7. Similar to Fig. 6, but for oblate cylinders. 
parameter of the spheroid is larger by a factor of three than that handled by the EBCM. To obtain a better knowledge of the accuracy of the geometric-optics method for smaller size parameters, the reader is referred to the comparison of the complete phase matrix elements of a randomly oriented spheroid simulated from the conventional geometric optics method (CGOM) and the $\mathrm{EBCM}$ in [34,53], or the IGOM and the EBCM in [14].

The comparisons of the phase matrix elements simulated from the IIM+SOV and the IGOM of prolate and oblate cylinders are presented in Figs. 6 and 7, respectively. The long-to-short-dimension ratio is 2 , and the size parameter defined in terms of half of the long dimension is 300 . The maximum convergent size parameter for an oblate cylinder of the extended-precision EBCM at this aspect ratio is 70 [50]. The excellent agreement between the IIM+SOV and the IGOM suggests the high reliability of the geometric optics method for large size parameter ranges. The differences between the IGOM and IIM+SOV for spheroids are relatively larger than those for cylinders. One possible explanation is the local radius of curvature on a cylindrical surface is much larger than on spheroidal surface and better fits the conditions of validity of the eikonal approximation for geometric optics. For cylinders, the radii of curvature on the top and bottom faces and the curves on side faces along the height are infinite, and the radius of curvature of the circular cross section is proportional to the size parameter. However, for spheroids, the radius of curvature is largest at the center and decreases as either end is approached.

\subsection{Comparison with discrete-dipole-approximation method: extreme cylinders, inhomogeneous particles, and aggregates}

We have shown the IIM+SOV method to be applicable to high aspect ratio and large size parameter cylindrical particles, which are out of the current computational range of the EBCM. For the purpose of comparison, we simulated the same scattering cases by using the DDA method on parallel computer clusters. To facilitate the DDA simulation for large size parameters, we considered a fixed orientation and the direction of the incident light aligned with the symmetry axis. Fig. 8 shows the comparison of
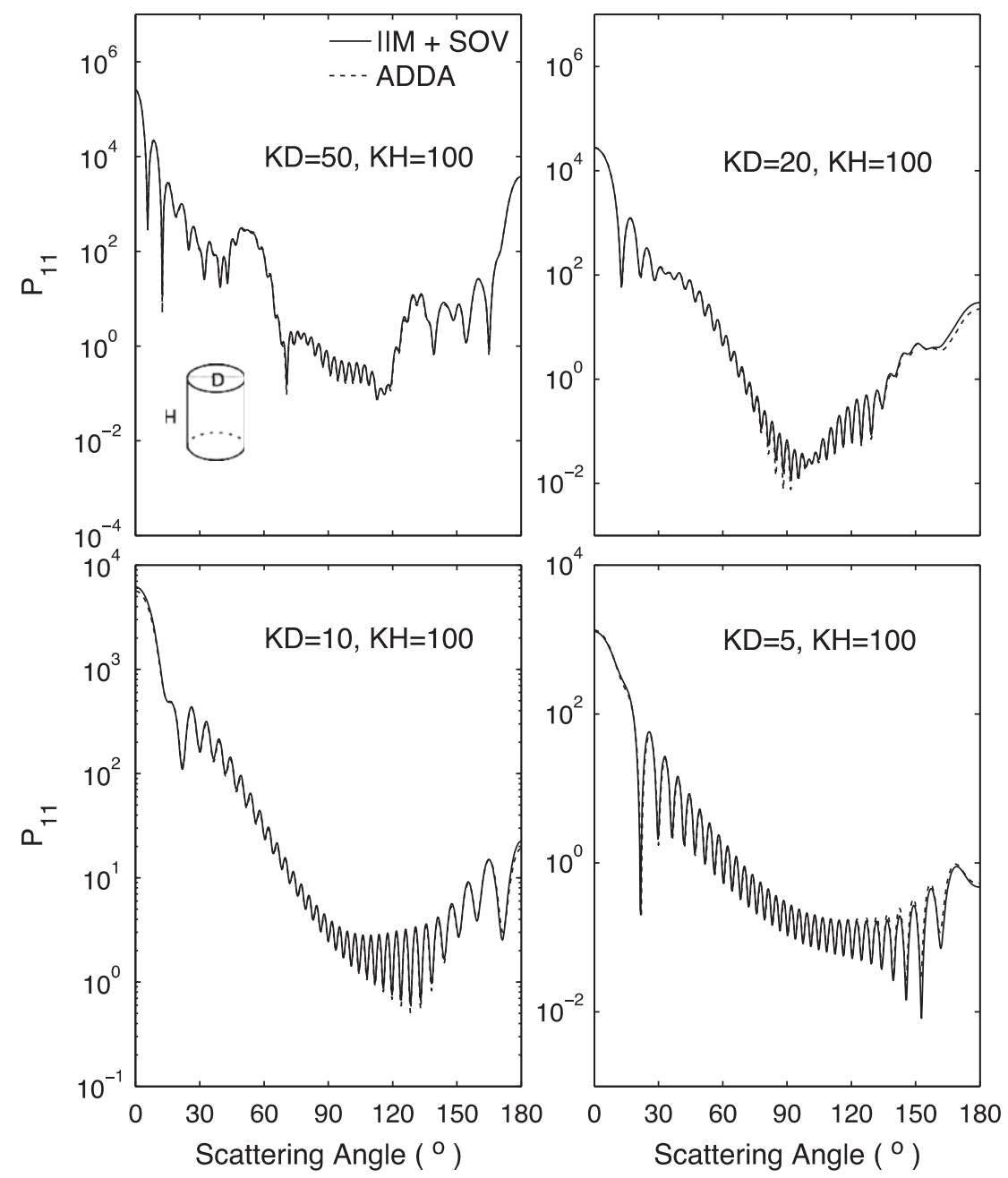

Fig. 8. $P_{11}$ elements computed from the ADDA and the IIM+SOV for cylinders of large aspect ratios where the standard EBCM fails. 

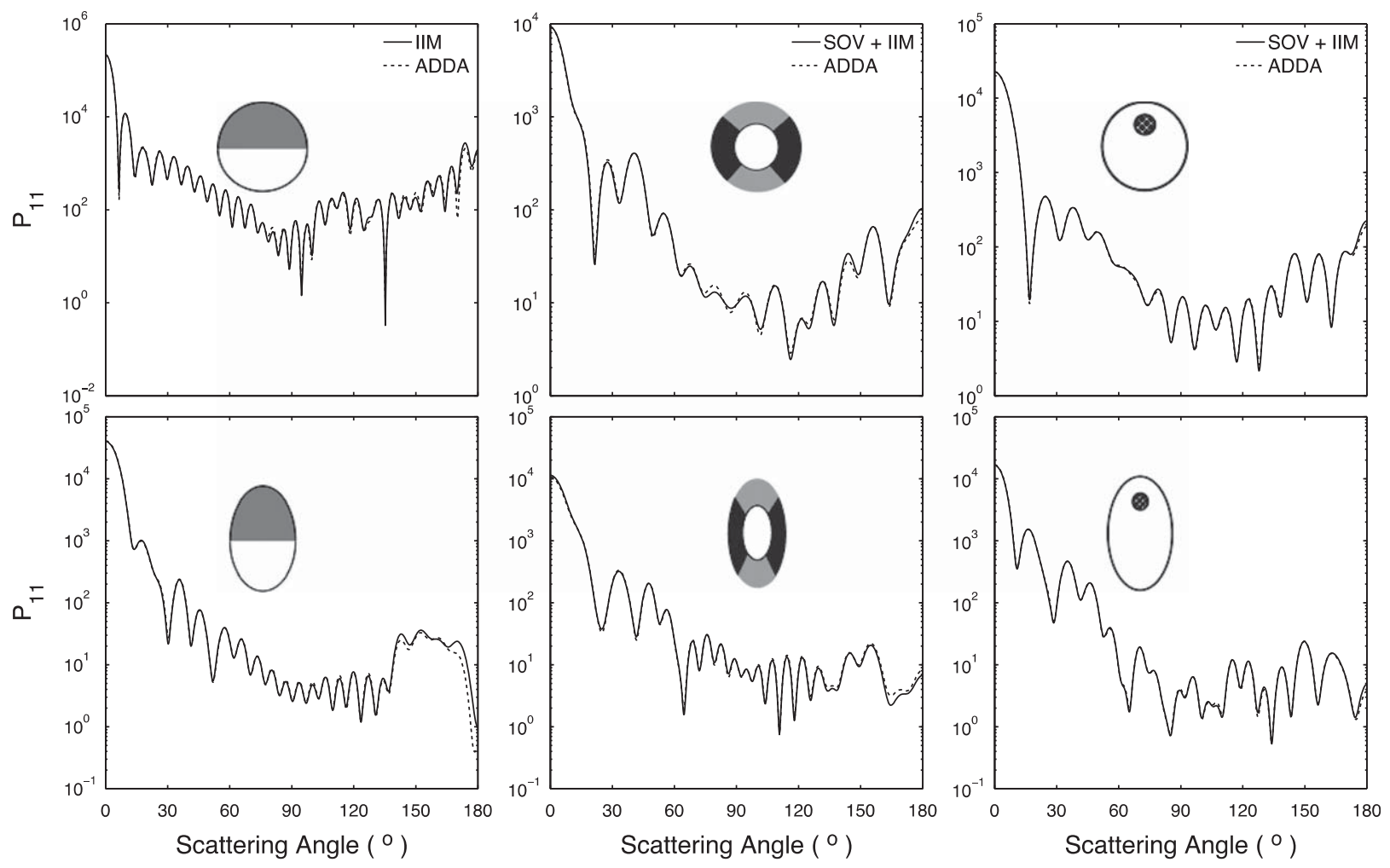

Fig. 9. Phase functions for six types of inhomogeneous particles.

the phase-matrix elements for circular cylinders of various aspect ratios simulated from the ADDA and the IIM+SOV. The fundamentally different algorithms give almost the same results. The advantage of the T-matrix over the ADDA is the computational efficiency of the IIM+SOV in cases of random orientations and large size parameters.

The computation of the T-matrix from the IIM+SOV has been demonstrated to be robust in handling light scattering by nonspherical particles. In addition to nonsphericity, natural particles often have heterogeneous compositions. For example, Asian dust aerosols originating in desert source regions can be coated by sulfates or soot when passing through heavily polluted downwind industrial regions [54], and Saharan dust grains transported over the Atlantic Ocean are often coated by sea salt.

As illustrative examples, we considered six types of representative inhomogeneous particles whose phase functions are shown in Fig. 9. In the first column, the size parameters of a sphere (defined in terms of its radius) and a prolate spheroid of aspect ratio 2 (defined in terms of its semi-major axis) are 30 . The refractive indices of the lower and upper part are 1.33 and 1.53. In the second column, the size parameter of a sphere (defined in terms of radius) is 15 and a prolate spheroid of aspect ratio 2 (defined in terms of its semi-major axis) is 30 . The ratio of the size parameters of the outer and inner radii is 0.8. The azimuthal angles necessary to separate different compositions are $30^{\circ}$ and $150^{\circ}$. The core refractive index is 1.53 , the black part is 1.33 , and the gray part is 1.70. Except for the refractive index, the size parameter and geometries in the third column are the same as their counterparts in the second column. The size parameter of the spherical inclusion is 2.5 in the sphere, and 7.5 in the spheroid. The distance between the center of inclusion and the host particle is 10 . In the simulations, the refractive index of the host particle is 1.33 and of the inclusion is 1.53. In the ADDA simulations, the more inhomogeneous the particle the slower the convergence, and to guarantee numerical accuracy, the number of dipoles per wavelength needs to be larger. Note that light scattering by a sphere with an inclusion was solved by Videen et al. [55] using an extension of Lorenz-Mie theory. The advantage of the IIM+SOV method is the simplicity with which it deals with diverse inhomogeneous particles.

Natural particulate matter has a tendency to aggregate and form single nonspherical particles. Scattering calculations for multiple nonspherical particles have been reported [56-59] based on the STMM. Fig. 10 shows phase functions of several two-particle systems computed by the IIM+SOV method and the ADDA. The shaded component represents a different refractive index from the other component. In the first column, we use the IIM to compute the T-matrix, although the IIM+SOV can be employed if the center of the particle geometry is shifted. In the remaining cases, we use the IIM+SOV because the inscribed sphere is easily identified. Note that for the bisphere and bi-prolate-spheroid systems, it is possible to compute the T-matrix for individual components and to use the STMM to obtain the T-matrix of the cluster. However, for the case shown in the third column, the superposition STMM fails because the circumscribed 

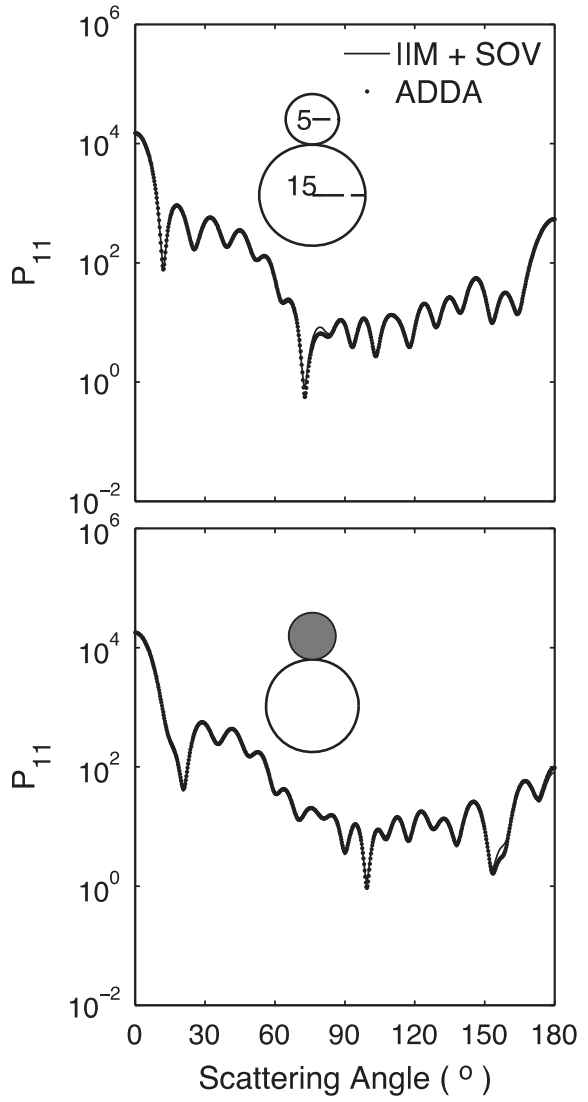
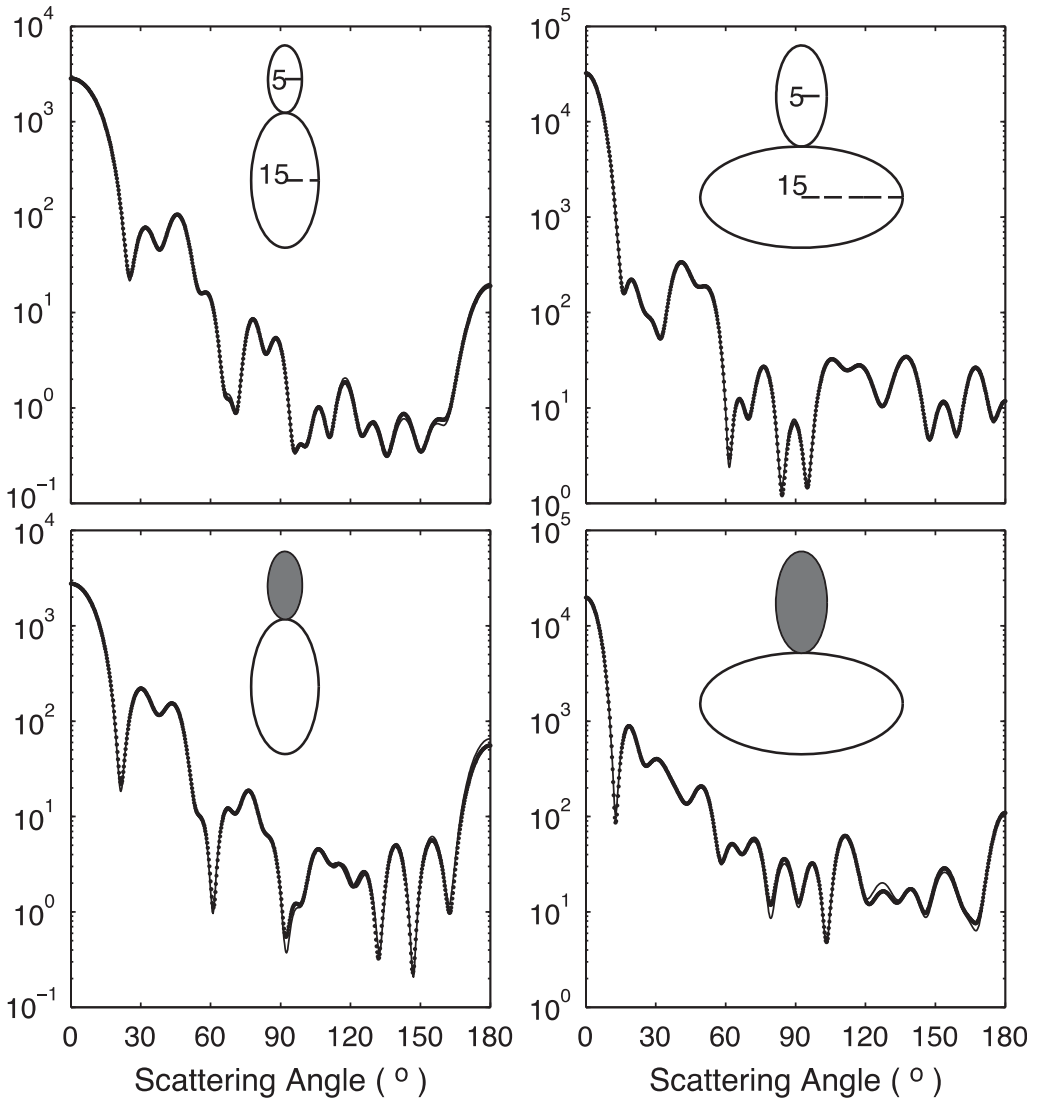

Fig. 10. Phase functions computed from the IIM+SOV and the ADDA for geometries indicated within the figure.

spheres of the two spheroids overlap [21]. To compute the phase function as well as for simplicity, we consider the direction of the incident light to be aligned with the symmetry axis. A very good agreement is demonstrated. The size parameters are indicated in the upper panel. The aspect ratios for the spheroids (major axis/minor axis) are assumed to be 2 , and the refractive index is 1.33 . The geometry and size of the particles in the lower panel are the same as those in the upper panels except that the refractive index in the shaded areas is 1.53 .

\section{Concluding remarks}

The IIM + SOV method provides an alternative approach to solving light scattering by nonspherical inhomogeneous particles. As compared with the EBCM, Johnson's IIM method had received less attention, and its modeling capabilities had not been extensively explored. In addition to the theoretical formulation, we have provided physical interpretation of terms in the iterative T-matrix equation. In this study, the IIM+SOV method has been applied to challenging or unprecedented computational domains, such as large size parameters, extreme geometries, and densely packed nonspherical particles.

The comparisons of the results simulated from the IIM+SOV, the EBCM, and the ADDA methods have illustrated that the present numerical implementation of the $\mathrm{IIM}+\mathrm{SOV}$ is correct. The simulated results satisfy the inherent relations, summarized by van Der Mee and Hovenier [49], with acceptable accuracy. However, a more accurate quantification of the applicability domain of the method for miscellaneous particles is still necessary. For example, for size parameters into the thousands, the algorithm must be parallelized according to a shared memory model. The extension of the IIM+SOV method to particles that lack axial symmetry is may also be a future research topic.

Compared to the EBCM, the particle morphology in the computational program is profoundly easier to modify, especially in the case of layered and composite particles. To further enhance the efficiency and the numerical capabilities of the IIM+SOV T-matrix method, an integration of the IIM, the EBCM, the SOV, and the superposition T-matrix method has been developed, and relevant results will be published in a separate paper.

\section{Acknowledgments}

This research is supported by the National Science Foundation (ATMO-0803779), NASA (NNX11AK37G), and the endowment funds associated with the David Bullock Harris Chair in Geosciences at the College of Geosciences, Texas A\&M University. The numerical computation was carried out using the EOS supercomputer at Texas A\&M University. MIM acknowledges support via the NASA 
Remote Sensing Theory Program managed by Lucia Tsaoussi. The authors thank M. A. Yurkin and A. G. Hoekstra for use of their ADDA code.

\section{Appendix A. Iterative T-matrix equations for inhomogeneous spherical layers}

The electromagnetic volume integral equation as given in Refs. $[7,21,60]$, reads

$$
\vec{E}(\vec{r})=\overrightarrow{E^{i n c}}(\vec{r})+k^{2} \int\left(\tilde{m}^{2}-1\right) \stackrel{\leftrightarrow}{G}\left(\vec{r}-\overrightarrow{r^{\prime}}\right) \vec{E}\left(\overrightarrow{r^{\prime}}\right) d^{3} \overrightarrow{r^{\prime}}
$$

where $\overrightarrow{E^{i n c}}$ is the incident electric field and $\stackrel{G}{G}\left(\vec{r}-\overrightarrow{r^{\prime}}\right)$ is the dyadic Green's function [48]:

$\overleftrightarrow{G}=\left(\overleftrightarrow{I}+\frac{1}{k^{2}} \vec{\nabla} \vec{\nabla}\right) \frac{\exp \left(i k\left|\vec{r}-\overrightarrow{r^{\prime}}\right|\right)}{4 \pi\left|\vec{r}-\overrightarrow{r^{\prime}}\right|}$

Let $\left|\vec{r}-\overrightarrow{r^{\prime}}\right|=R$; then the explicit expression for $\overleftrightarrow{G}$ is given by

$\overleftrightarrow{G}\left(\vec{r}-\overrightarrow{r^{\prime}}\right)=\frac{\exp (i k R)}{4 \pi R}\left[\left(\overleftrightarrow{I}-\frac{\vec{R} \vec{R}}{R^{2}}\right)-\frac{1-i k R}{(k R)^{2}}\left(\overleftrightarrow{I}-\frac{3 \vec{R} \vec{R}}{(k R)^{2}}\right)\right]$

The singularity term associated with the $1 / R^{3}$ dependence in Eq. (41) is as follows,

$\overleftrightarrow{G}^{s}=\frac{1}{4 \pi k^{2}} \vec{\nabla} \vec{\nabla} \frac{1}{R}=\frac{1}{4 \pi R}\left[\frac{-1}{k^{2} R^{2}}-\hat{R} \hat{R} \frac{3}{k^{2} R^{2}}\right]$,

where the cap denotes the unit vector in a specific direction. To treat the singularity, Eq. (39) can be written as

$$
\begin{aligned}
\vec{E}(\vec{r})= & \vec{E}_{\text {inc }}(\vec{r})+k^{2} \int\left(\tilde{m}^{2}-1\right)\left[\overleftrightarrow{G}\left(\vec{r}-\vec{r}^{1}\right)\right. \\
& \left.+\frac{\hat{r} \hat{r}}{k^{2}} \delta(\vec{r}-\vec{r})\right] \vec{E}(\vec{r}) d^{3} \overrightarrow{r^{\prime}},
\end{aligned}
$$

where $\overleftrightarrow{G}\left(r-\vec{r}^{1}\right)$ is the Green function valid at the source free regions. Eq. (43) is equivalent to

$$
\overrightarrow{E^{e f f}}(\vec{r})=\overrightarrow{E^{i n c}}(\vec{r})+\int_{v} d^{3} \vec{r} \overleftrightarrow{G}\left(\vec{r}, \overrightarrow{r^{\prime}}\right)\left[\tilde{m}\left(\overrightarrow{r^{\prime}}\right)^{2}-1\right] \overleftrightarrow{Z}\left(\overrightarrow{r^{\prime}}\right) \overrightarrow{E^{e f f}}\left(\overrightarrow{r^{\prime}}\right)
$$

where $\overrightarrow{E^{e f f}}\left(\overrightarrow{r^{\prime}}\right)$ is the total field in source free regions, and $\overleftrightarrow{Z}\left(r^{\prime}\right)[40]$ is a Cartesian tensor given by

$\overleftrightarrow{Z}\left(\overrightarrow{r^{\prime}}\right)=1 / \tilde{m}^{2} \hat{r} \hat{r}+\hat{\theta} \hat{\theta}+\hat{\phi} \hat{\phi}$.

In the present context of a spherical coordinate system, it is preferable to classify the light scattering by particles into two groups: homogenous spheres and inhomogeneous spheres. The scattering by nonspherical particles is equivalent to that by an inhomogeneous circumscribed sphere (the refractive index of the space between the spherical surface and the particle space is different from that inside the particle). On the basis of Eq. (7), the dyadic Green's function in the matrix form is written as

$\overline{\overline{\mathbf{G}}}_{0}\left(\vec{r}, \overrightarrow{r^{\prime}}\right)=\sum_{n=1}^{\infty} \sum_{m=-n}^{n} \overline{\overline{\mathbf{Y}}}_{m n}(\theta, \phi) \overline{\overline{g_{n}}}\left(\vec{r},{\overrightarrow{r^{\prime}}}^{\prime} \overline{\overline{\mathbf{Y}}}_{m n}^{T}\left(\theta^{\prime}, \phi^{\prime}\right)\right.$,

where $g_{n}\left(\vec{r}, \overrightarrow{r^{\prime}}\right)$ is a 3-by-3 matrix given by Eq. (25).
Substituting Eqs. (46) and (25) into Eq. (44), we obtain

$$
\begin{aligned}
& \overline{\overline{\mathbf{E}}}_{m^{\prime} n^{\prime}}(\vec{r})=\overline{\overline{\mathbf{Y}}}_{m^{\prime} n^{\prime}}(\theta, \phi) \overline{\overline{\mathbf{J}}}_{n^{\prime}}(r) \\
& \quad+\int_{v} d r^{\prime} r^{\prime 2} d \Omega^{\prime} \sum_{n=1}^{\infty} \sum_{m=-n}^{n} \overline{\overline{\mathbf{Y}}}_{m n}(\theta, \phi) g_{n}\left(r, r^{\prime}\right) \mathbf{Y}_{m n}^{T, *}\left(\theta^{\prime}, \phi^{\prime}\right) u\left(\overrightarrow{r^{\prime}}\right) \overline{\overline{\mathbf{Z}}}\left(\vec{r}^{\prime}\right) \overline{\overline{\mathbf{E}}}_{m^{\prime} n^{\prime}}(\vec{r}),
\end{aligned}
$$

and in a simplified form

$\overline{\overline{\mathbf{E}}}_{m^{\prime} n^{\prime}}(\vec{r})=\overline{\overline{\mathbf{Y}}}_{m^{\prime} n^{\prime}}(\theta, \phi) \overline{\overline{\mathbf{J}}}_{n^{\prime}}(r)+\int_{0}^{R} d r^{\prime} \sum_{n=1}^{\infty} \sum_{m=-n}^{n} \overline{\overline{\mathbf{Y}}}_{m^{\prime} n^{\prime}}(\theta, \phi) g_{n}\left(r, r^{\prime}\right) \overline{\overline{\mathbf{F}}}_{m n m^{\prime} n^{\prime}}\left(r^{\prime}\right)$

$\overline{\bar{F}}_{m n m^{\prime} n^{\prime}}(\vec{r})=r^{2} \int_{v} d \Omega^{\prime} \overline{\bar{Y}}_{m n}^{T, *}\left(\theta^{\prime}, \phi^{\prime}\right) u(\vec{r}) \overline{\bar{Z}}(\vec{r}) E_{m^{\prime} n^{\prime}}(\vec{r})$

Substituting Eq. (48) into Eq. (49) yields

$$
\begin{aligned}
\overline{\overline{\mathbf{F}}}_{m n m^{\prime} n^{\prime}}(r)= & \overline{\overline{\mathbf{U}}}_{m n m^{\prime} n^{\prime}}(r) \overline{\overline{\mathbf{J}}}_{n^{\prime}}(r) \\
& +\int_{0}^{R} d r^{\prime} \sum_{\tilde{n}=1}^{\infty} \sum_{\tilde{m}=-\tilde{n}}^{n} \overline{\overline{\mathbf{U}}}_{m n, \tilde{m} \tilde{n}}(r) g_{\tilde{n}}\left(r, r^{\prime}\right) \overline{\overline{\mathbf{F}}}_{\tilde{m} \tilde{n} m^{\prime} n^{\prime}}\left(r^{\prime}\right)
\end{aligned}
$$

where

$\overline{\overline{\mathbf{U}}}_{m n m^{\prime} n^{\prime}}(r)=r^{2} \int d \Omega\left[\overline{\mathbf{Y}}_{m n}(\theta, \phi)\right]^{T *} u(r) \overline{\overline{\mathbf{Z}}}(r) \overline{\mathbf{Y}}_{m^{\prime} n^{\prime}}(\theta, \phi)$.

Based on Eq. (48), the T-matrix is written as,

$\overline{\bar{T}}_{m n m^{\prime} n^{\prime}}(\vec{r})=i k \int_{0}^{R} d r^{\prime} \overline{\bar{J}}_{n}^{T}\left(r^{\prime}\right) \overline{\bar{F}}_{m n m^{\prime} n^{\prime}}\left(r^{\prime}\right)$,

To numerically compute the T-matrix, the integral is represented as summations

$\overline{\overline{\mathbf{T}}}\left(r_{n}\right)=i k \sum_{j=1}^{n} w_{j} \overline{\overline{\mathbf{J}}}\left(r_{j}\right) \overline{\overline{\mathbf{F}}}\left(n \mid r_{j}\right)$,

$\overline{\overline{\mathbf{F}}}\left(n \mid r_{i}\right)=\overline{\overline{\mathbf{U}}}\left(r_{i}\right) \overline{\overline{\mathbf{J}}}\left(r_{i}\right)+\sum_{j=1}^{n} w_{j} \overline{\overline{\mathbf{U}}}\left(r_{i}\right) \overline{\bar{g}}\left(r_{i}, r_{j}\right) \overline{\overline{\mathbf{F}}}\left(n \mid r_{j}\right)$.

Based on Eq. (54), we have

$$
\begin{aligned}
\overline{\overline{\mathbf{F}}}\left(n \mid r_{n}\right)= & \overline{\overline{\mathbf{U}}}\left(r_{n}\right) \overline{\overline{\mathbf{J}}}\left(r_{n}\right)+\sum_{j=1}^{n-1} w_{j} \overline{\overline{\mathbf{U}}}\left(r_{n}\right) \overline{\bar{g}}\left(r_{n}, r_{j}\right) \overline{\overline{\mathbf{F}}}\left(n \mid r_{j}\right) \\
& +w_{n} \overline{\overline{\mathbf{U}}}\left(r_{n}\right) \overline{\bar{g}}\left(r_{n}, r_{n}\right) \overline{\overline{\mathbf{F}}}\left(n \mid r_{n}\right),
\end{aligned}
$$

and

$$
\begin{aligned}
& {\left[\overline{\overline{\mathbf{I}}}-w_{n} \overline{\overline{\mathbf{U}}}\left(r_{n}\right) \overline{\bar{g}}\left(r_{n}, r_{n}\right)\right] \overline{\overline{\mathbf{F}}}\left(n \mid r_{n}\right)=\overline{\overline{\mathbf{U}}}\left(r_{n}\right)} \\
& \quad \times\left[\overline{\overline{\mathbf{J}}}\left(r_{n}\right)+\sum_{j=1}^{n-1} w_{j} \overline{\bar{g}}\left(r_{n}, r_{j}\right) \overline{\overline{\mathbf{F}}}\left(n \mid r_{j}\right)\right] .
\end{aligned}
$$

Inserting Eq. (25) into Eq. (56) gives

$$
\begin{aligned}
\overline{\overline{\mathbf{F}}}\left(n \mid r_{n}\right)= & w_{n}{ }^{-1} w_{n} \frac{\overline{\overline{\mathbf{U}}}\left(r_{n}\right)}{\left[I-w_{n} \overline{\overline{\mathbf{U}}}\left(r_{n}\right) \overline{\bar{g}}\left(r_{n}, r_{n}\right)\right]} \\
& \times\left[\overline{\overline{\mathbf{J}}}\left(r_{n}\right)+\overline{\overline{\mathbf{H}}}\left(r_{n}\right) i k \sum_{j=1}^{n-1} w_{j} \tilde{J}\left(r_{j}\right) F\left(n \mid r_{j}\right)\right] .
\end{aligned}
$$

Define

$\overline{\overline{\mathbf{Q}}}=\frac{w_{n} \overline{\overline{\mathbf{U}}}\left(r_{n}\right)}{\left[\overline{\overline{\mathbf{I}}}-w_{n} \overline{\overline{\mathbf{U}}}\left(r_{n}\right) \overline{\bar{g}}\left(r_{n}, r_{n}\right)\right]}, \quad \overline{\bar{q}}=i k \sum_{j=1}^{n-1} w_{j} \overline{\overline{\mathbf{J}^{T}}}\left(r_{j}\right) \overline{\overline{\mathbf{F}}}\left(n \mid r_{j}\right)$, 
then

$\overline{\overline{\mathbf{F}}}\left(n \mid r_{n}\right)=w_{n}{ }^{-1} \overline{\overline{\mathbf{Q}}}\left[\overline{\bar{J}}\left(r_{n}\right)+\overline{\overline{\mathbf{H}}}\left(r_{n}\right) \overline{\bar{q}}\right]$.

Based on Eqs. (53),(58) and (59), we obtain

$$
\begin{aligned}
\overline{\overline{\mathbf{T}}}\left(r_{n}\right) & =i k \sum_{j=1}^{n-1} w_{j} \overline{\overline{\mathbf{J}^{T}}}\left(r_{j}\right) \overline{\overline{\mathbf{F}}}\left(n \mid r_{j}\right)+i k w_{n} \overline{\overline{\mathbf{J}^{T}}}\left(r_{n}\right) \overline{\overline{\mathbf{F}}}\left(n \mid r_{n}\right) \\
& =\overline{\bar{q}}+i k w_{n} \overline{\overline{\mathbf{J}^{T}}}\left(r_{n}\right) \overline{\overline{\mathbf{F}}}\left(n \mid r_{n}\right) \\
& =\overline{\bar{q}}+i k \overline{\overline{\mathbf{J}^{T}}}\left(r_{n}\right) \overline{\overline{\mathbf{Q}}}\left[\overline{\overline{\mathbf{J}}}\left(r_{n}\right)+\overline{\overline{\mathbf{H}}}\left(r_{n}\right) \overline{\bar{q}}\right] \\
& =\overline{\bar{q}}+\overline{\overline{\mathbf{Q}}}_{11}+\overline{\overline{\mathbf{Q}}}_{12} \overline{\bar{q}} \\
& =\overline{\overline{\mathbf{Q}}}_{11}+\left(\overline{\overline{\mathbf{I}}}+\overline{\overline{\mathbf{Q}}}_{12}\right) \overline{\bar{q}},
\end{aligned}
$$

where $\overline{\overline{\mathbf{Q}}}_{1,1}=i k \overline{\overline{\mathbf{J}}} \overline{\overline{\mathbf{Q}}} \overline{\overline{\mathbf{J}}}$ and $\overline{\mathbf{Q}}_{1,2}=i k \overline{\overline{\mathbf{J}}} \overline{\overline{\mathbf{Q}}} \overline{\overline{\mathbf{H}}}$. Beginning with the following equation,

$\overline{\overline{\mathbf{T}}}\left(r_{n-1}\right)=i k \sum_{j=1}^{n-1} w_{j} \overline{\overline{\mathbf{J}^{T}}}\left(r_{j}\right) \overline{\overline{\mathbf{F}}}\left(n-1 \mid r_{j}\right)$

let

$\overline{\overline{\mathbf{F}}}\left(n \mid r_{j}\right)=\overline{\overline{\mathbf{F}}}\left(n-1 \mid r_{j}\right)(\overline{\overline{\mathbf{I}}}+\overline{\bar{p}})$.

then

$\overline{\overline{\mathbf{T}}}\left(r_{n-1}\right)(\overline{\overline{\mathbf{I}}}+\overline{\bar{p}})=\overline{\bar{q}}$.

Based on Eqs. (54) and (62), we have

$$
\begin{gathered}
\overline{\overline{\mathbf{F}}}\left(n-1 \mid r_{i}\right)(\overline{\overline{\mathbf{I}}}+\overline{\bar{p}})=\overline{\overline{\mathbf{U}}}\left(r_{i}\right) \overline{\overline{\mathbf{J}}}\left(r_{i}\right)+\sum_{j=1}^{n-1} w_{j} \overline{\overline{\mathbf{U}}}\left(r_{i}\right) \overline{\bar{g}}\left(r_{i}, r_{j}\right) \\
\times \overline{\overline{\mathbf{F}}}\left(n-1 \mid r_{j}\right)(\overline{\overline{\mathbf{I}}}+\overline{\bar{p}})+w_{n} \overline{\overline{\mathbf{U}}}\left(r_{i}\right) \overline{\bar{g}}\left(r_{i}, r_{n}\right) \overline{\overline{\mathbf{F}}}\left(n \mid r_{n}\right)
\end{gathered}
$$

Note that Eq. (54) is satisfied if $n$ is replaced with $n-1$, and Eq. (64) is in the following form

$\overline{\overline{\mathbf{U}}}\left(r_{i}\right) \overline{\overline{\mathbf{J}}}\left(r_{i}\right) \overline{\bar{p}}=\overline{\overline{\mathbf{U}}}\left(r_{i}\right) \overline{\bar{g}}\left(r_{i}, r_{n}\right) \overline{\overline{\mathbf{Q}}}\left[\overline{\overline{\mathbf{J}}}\left(r_{n}\right)+\overline{\overline{\mathbf{H}}}\left(r_{n}\right) \overline{\bar{q}}\right]$.

Comparing Eqs. (25) and (65), we obtain

$\overline{\bar{p}}=\overline{\overline{\mathbf{Q}}}_{2,1}+\overline{\overline{\mathbf{Q}}}_{2,2} \overline{\bar{q}}$

Combining Eqs. (63) and (66), we have

$\overline{\overline{\mathbf{T}}}\left(r_{n-1}\right)\left(\overline{\overline{\mathbf{I}}}+\overline{\overline{\mathbf{Q}}}_{2,1}+\overline{\overline{\mathbf{Q}}}_{2,2} \overline{\bar{q}}\right)=\overline{\bar{q}}$

where $\overline{\overline{\mathbf{Q}}}_{2,1}=i k \overline{\overline{\mathbf{H}^{T}}} \overline{\overline{\mathbf{Q}}} \overline{\mathbf{J}}$, and $\overline{\overline{\mathbf{Q}}}_{2,2}=i k \overline{\overline{\mathbf{H}^{T}}} \overline{\overline{\mathbf{Q}}} \overline{\overline{\mathbf{H}}}$. Solving Eq. (67) in terms of $\overline{\bar{q}}$,

$\overline{\bar{q}}=\left[\overline{\overline{\mathbf{I}}}-\overline{\overline{\mathbf{T}}}\left(r_{n-1}\right) \overline{\overline{\mathbf{Q}}}_{2,2}\right]^{-1} \overline{\overline{\mathbf{T}}}\left(r_{n-1}\right)\left(\overline{\overline{\mathbf{I}}}+\overline{\overline{\mathbf{Q}}}_{2,1}\right)$.

Substituting Eq. (68) into Eq. (60), we have Eq. (17), which is the final iterative formula to calculate the T-matrix. The information about particle shape and composition is contained in the U-matrix given by Eq. (23). Note that the definition of the U-matrix (relevant quantities) is different if VSWFs are formulated based on odd and even modes [40], although the same symbols are used.

\section{References}

[1] Mie G. Beiträge zur Optik trüber Medien, speziell kolloidaler Metallösungen. Ann Phys 1908;25:377-445.

[2] van de Hulst HC. Light scattering by small particles. New York: Dover; 1981.
[3] Wiscombe WJ. Improved Mie scattering algorithms. Appl Opt 1980;19:1505-9.

[4] Bohren CF, Huffman DR. Absorption and scattering of light by small particles. New York: Wiley; 1983.

[5] Liu L, Wang H, Yu B, Xua Y, Shen J. Improved algorithm of light scattering by a coated sphere. China Particuology 2007;5:230-6.

[6] Mishchenko MI, Hovenier JW, Travis LD. Light scattering by nonspherical particles: theory, measurements, and applications. San Diego: Academic Press; 1999.

[7] Kahnert FM. Numerical methods in electromagnetic scattering theory. J Quant Spectrosc Radiat Trans 2003;79-80:775-824.

[8] Mishchenko MI, Travis LD. T-matrix computations of light scattering by large spheroidal particles. Opt Commun 1994;109:16-21.

[9] Yurkin MA, Hoekstra AG. The discrete-dipole-approximation code ADDA: capabilities and known limitations. J Quant Spectrosc Radiat Trans 2011;112:2234-47.

[10] Mackowski DW, Mishchenko MI. A multiple sphere T-matrix FORTRAN code for use on parallel computer clusters. J Quant Spectrosc Radiat Trans 2011;112:2182-92.

[11] Liu C, Panetta RL, Yang P. Application of the pseudo-spectral time domain method to compute particle single-scattering properties for size parameters up to 200. J Quant Spectrosc Radiat Trans 2012;113:1728-40.

[12] Bi L, Yang P, Kattawar GW, Hu Y, Baum BA. Scattering and absorption of light by ice particles: solution by a new physicalgeometric optics hybrid method. J Quant Spectrosc Radiat Trans 2011;112:1492-508.

[13] Liou KN, Takano Y, Yang P. Light absorption and scattering by aggregates: application to black carbon and snow grains. J Quant Spectrosc Radiat Trans 2011;112:1581-94.

[14] Yang P, Feng Q, Hong G, Kattawar GW, Wiscombe WJ, Mishchenko $\mathrm{MI}$, et al. Modeling of the scattering and radiative properties of nonspherical dust-like aerosols. J Aerosol Sci 2007;38:995-1014.

[15] Bi L, Yang P, Kattawar GW, Kahn R. Single-scattering properties of triaxial ellipsoidal particles for a size parameter range from the Rayleigh to geometric-optics regimes. Appl Opt 2009;48:114-26.

[16] Bi L, Yang P, Kattawar GW, Kahn R. Modeling optical properties of mineral aerosol particles by using nonsymmetric hexahedra. Appl Opt 2010;49:334-42.

[17] Waterman PC. Matrix formulation of electromagnetic scattering. Proceedings of IEEE 1965;53:805-12.

[18] Waterman PC. Symmetry, unitarity, and geometry in electromagnetic scattering. Phys Rev D 1971;3:825-39.

[19] Barber P, Yeh C. Scattering of electromagnetic waves by arbitrarily shaped dielectric bodies. Appl Opt 1975;14:2864-72.

[20] Barber PW, Hill SC. Light scattering by particles: computational methods. Singapore: World Scientific; 1990.

[21] Mishchenko MI, Travis LD, Lacis AA. Scattering, absorption and emission of light by small particles. Cambridge: Cambridge University Press; 2002.

[22] Doicu A, Wriedt T, Eremin Y. Light scattering by systems of particles. Berlin, Heidelberg, New York: Springer; 2006.

[23] Yee SK. Numerical solution of initial boundary value problems involving Maxwell's equations in isotropic media. IEEE Trans Antennas Propag 1966;14:302-7.

[24] Yang P, Liou KN. Finite-difference time domain method for light scattering by small ice crystals in three-dimensional space. J Opt Soc Am A 1996;13:2072-85.

[25] Sun W, Fu Q Chen Z. Finite-difference time-domain solution of light scattering by dielectric particles with perfectly matched layer absorbing boundaryconditions. Appl Opt 1999;38:3141-51.

[26] Purcell EM, Pennypacker CR. Scattering and absorption of light by nonspherical dielectric grains. Astrophys J 1973;186:705-14.

[27] Draine BT, Flatau PJ. Discrete-dipole approximation for scattering calculations. J Opt Soc Am A 1994;11:1491-9.

[28] Yurkin MA, Maltsev VP, Hoekstra AG. The discrete dipole approximation for simulation of light scattering by particles much larger than the wavelength. J Quant Spectrosc Radiat Trans 2007;106:546-57.

[29] Mishchenko MI. Light scattering by randomly oriented axially symmetric particles. J Opt Soc Am A 1991;8:871-82.

[30] Peterson B, Ström S. T matrix for electromagnetic scattering from an arbitrary number of scatterers and representations of $\mathrm{E}(3) *$. Phys Rev D 1973:8:3661-78.

[31] Peterson B, Ström S. T-matrix formulation of electromagnetic scattering from multilayered scatterers. Phys Rev D 1974;10: $2670-84$

[32] Ström SW, Zheng W. The null field approach to electromagnetic scattering from composite objects. IEEE Trans Antennas Propag $1988 ; 36: 376-82$ 
[33] Borghese F, Denti P, Saija R. Scattering from model nonspherical particles. Berlin: Springer; 2007.

[34] Wielaard DJ, Mishchenko MI, Macke A, Carlson BE. Improved Tmatrix computations for large, nonabsorbing and weakly absorbing nonspherical particles and comparison with geometrical-optics approximation. Appl Opt 1997;36:4305-13.

[35] Yan WZ, Du Y, Wu H, Liu DW. EM scattering from a long dielectric circular cylinder. Prog Electromagn Res 2008;85:39-67.

[36] Kahnert M, Rother T. Modeling optical properties of particles with small-scale surface roughness: combination of group theory with a perturbation approach. Opt Express 2011;19:11138-51.

[37] Somerville WRC, Auguie B, Le Ru EC. Severe loss of precision in calculation of T-matrix integrals. J Quant Spectrosc Radiat Transfer 2012;113:524-35.

[38] Mishchenko MI, Zakharova NT, Videen G, Khlebtsov NG, Wriedt T. Comprehensive T-matrix reference database: a 2007-2009 update. J Quant Spectrosc Radiat Transfer 2010;111:650-8.

[39] Zakharova NT, Videen G, Khlebtsov NG. Comprehensive T-matrix reference database: a 2009-2011 update. J Quant Spectrosc Radiat Transfer 2012:113:1844-52.

[40] Johnson BR. Invariant imbedding T-matrix approach to electromagnetic scattering. Appl Opt 1988;27:4861-73.

[41] Schulz FM, Stamnes K, Stamnes JJ. Scattering of electromagnetic waves by spheroidal particles: a novel approach exploiting the $\mathrm{T}$ matrix computed in spheroidal coordinates. Appl Opt 1998;37: 7875-96.

[42] Mackowski DW. Discrete dipole moment method for calculation of the T matrix for nonspherical particles. J Opt Soc Am A 2002;19: 881-93.

[43] Loke VLY, Nieminen TA, Heckenberg NR, Rubinsztein-Dunlop H. Tmatrix calculation via discrete-dipole approximation, point matching and exploiting symmetry. J Quant Spectrosc Radiat Transf 2009;110:1460-71.

[44] Mackowski DW, Mishchenko MI. Calculation of the T matrix and the scattering matrix for ensembles of spheres. J Opt Soc Am A 1996;13:2266-78.

[45] Johnson BR, Secrest D. Quantum mechanical calculations of the inelastic cross sections for rotational excitation of para and ortho $\mathrm{H}_{2}$ upon collision with He. J Chem Phys 1968;48:4682-93.
[46] Adams CN, Kattawar GW. Solutions of the equations of radiative transfer by an invariant imbedding approach. J Quant Spectrosc Radiat Transfer 1970;10:341-66.

[47] Mishchenko MI. Calculation of the amplitude matrix for a nonspherical particle in a fixed orientation. Appl Opt 2000;39:1026-31.

[48] Tsang L, Kong JA, Shin RT. Theory of microwave remote sensing. New York: Wiley; 1985

[49] van der Mee CVM, Hovenier JW. Expansion coefficients in polarized light transfer. Astron Astrophys 1990;228:559-68.

[50] Mishchenko MI, Travis LD. Capabilities and limitations of a current FORTRAN implementation of the T-matrix method for randomly oriented, rotationally symmetric scatterers. J Quant Spectrosc Radiat Transfer 1998;60:309-24.

[51] Mishchenko MI, Travis LD, Macke A. Scattering of light by polydisperse, randomly oriented, finite circular cylinders. Appl Opt 1996;35:4927-40.

[52] Mishchenko MI, Macke A. How big should hexagonal ice crystals be to produce halos? Appl Opt 1999;38:1626-9.

[53] Macke A, Mishchenko MI, Muinonen K, Carlson BE. Scattering of light by large nonspherical particles: ray tracing approximation versus T-matrix method. Opt Lett 1995;20:1934-6.

[54] Parungo FP, Nagamoto CT, Madel R, Rosinski J, Haagenson PL. Marine aerosols in Pacific upwelling regions. J Aerosol Sci 1987;18: 277-90.

[55] Videen G, Ngo D, Chýlek P, Pinnick RG. Light scattering from a sphere with an irregular inclusion. J Opt Soc Am A 1995;12:922-8.

[56] Xu YL. Scattering Mueller matrix of an ensemble of variously shaped small particles. J Opt Soc Am A 2003;20:2093-105.

[57] Jin YQ, Huang X. Numerical T-matrix solution for polarized scattering from a cluster of spatially oriented, nonspherical scatterers. Microwave Opt Technol Lett 1996;12:154-8.

[58] Liu L, Mishchenko MI. Scattering and radiative properties of complex soot and soot-containing aggregate particles. J Quant Spectrosc Radiat Transfer 2007;106:262-73.

[59] Liu L, Mishchenko MI, Arnott WP. A study of radiative properties of fractal soot aggregates using the superposition T-matrix method. J Quant Spectrosc Radiat Transfer 2008;109:2656-63.

[60] Lakhtakia A. Strong and weak forms of the method of moments and the coupled dipole method for scattering of time-harmonic electromagnetic fields. Int J Mod Phys C 1992;3:583-603. 